\begin{tabular}{|c|l|}
\hline Title & One cocycles on smooth flows of weights and extended modular coactions \\
\hline Author(s) & YAMA NOUCHI, TA KEHIKO \\
\hline Citation & $\begin{array}{l}\text { Ergodic Theory and Dynamical Systems, 27(1), 285-318 } \\
\text { https://doi.org/10.1017/S014338570600551 }\end{array}$ \\
\hline Issue Date & 2007-02 \\
\hline Doc URL & http://hdl.handle.net/2115/20159 \\
\hline Rights & Copyright $\odot 2007$ Cambridge University Press \\
\hline Type & article \\
\hline File Information & ETDS27-1.pdf \\
\hline
\end{tabular}

Instructions for use 
Ergod. Th. \& Dynam. Sys. (First published online 2006), 0, 1-34*

doi:10.1017/S0143385706000551 $\quad$ (C) 2006 Cambridge University Press

*Provisional-final page numbers to be inserted when paper edition is published

\title{
One-cocycles on smooth flows of weights and extended modular coactions
}

\author{
TAKEHIKO YAMANOUCHI \\ Department of Mathematics, Faculty of Science, Hokkaido University, \\ Sapporo 060-0810, Japan \\ (e-mail: yamanouc@math.sci.hokudai.ac.jp)
}

(Received 9 June 2005 and accepted in revised form 15 June 2006)

\section{Introduction}

This paper is concerned with Borel 1-cocycles on ergodic flows on standard Borel measure spaces and a certain type of group coactions on (separable) factors.

In [3], Aoi and the present author clarified a close relation between Borel 1-cocycles in ergodic theory and group coactions in the theory of operator algebras: it was proven there that cocycles on a discrete measured equivalence relation $\mathcal{R}$ are, roughly speaking, in bijective correspondence with coactions on the associated (Feldman-Moore) von Neumann algebra $W^{*}(\mathcal{R})$ (see [14]) which fix the Cartan subalgebra of $W^{*}(\mathcal{R})$ pointwise. This would provide a reasonable evidence that the study of 1-cocycles on $\mathcal{R}$ is 'equivalent' to that of special group coactions on $W^{*}(\mathcal{R})$, and thus enables us to analyze such cocycles by operator-algebraic methods and investigate coactions of the type described above from a viewpoint of ergodic theory.

Note that 1-cocycles on discrete equivalence relations are almost the same as those on measure spaces with actions of countable groups (see [13]). Hence, if we pursue the research along the line illustrated above, we are naturally led to consider cocycles on measure spaces with continuous group actions, especially cocycles on flow spaces. They are exactly what we treat in this paper.

Let $\left\{F_{t}\right\}_{t \in \mathbb{R}}$ be a (conservative) ergodic flow on a standard probability space $(X, \mu)$. We may think of the covariant system $\left(X, \mathbb{R},\left\{F_{t}\right\}\right)$ as the smooth flow of weights on an approximately finite-dimensional (AFD) factor $N$ of type III. Consider a Borel 1 -cocycle $c: \mathbb{R} \times X \rightarrow K$ on this ergodic $\mathbb{R}$-space $X$ with values in a (second countable) locally compact group $K$. If $K$ happens to be the one-dimensional torus $\mathbb{T}$, then, for each dominant weight $\phi$ on $N$, the cocycle $c$ induces a $*$-automorphism $\sigma_{c}^{\phi}$ of $N$, called the extended modular automorphism of $\phi$ (see [9]). Thanks to [9], we further know which automorphisms of $N$ arise in this manner.

Given this fact, one might ask what happens if $K$ is a general locally compact group. To the best of the author's knowledge, this problem was treated beautifully by Izumi [22] in a different context when $K$ is compact. To be more precise, he considered a factor $N$ of type III that admits a family of special (i.e. modular) endomorphisms of $N$ with a certain 
set of properties. Each member $\pi$ of this family induces a Borel 1-cocycle on the smooth flow of weights on $N$ with values in the unitary group $U(n)$, where $n$ depends on $\pi$. Due to one of the results of Zimmer in [40], the family then determines a compact group $K$. The properties which this family of endomorphisms enjoys now ensure that $N$ admits a Roberts action (equivalently, a coaction) of $K$.

Although we could not always hope that this kind of endomorphism exists on $N$ when $K$ is no longer a compact group, we might still expect that each 1-cocycle on the smooth flow of weights on $N$ induces a coaction of $K$ on $N$. The main purpose of this paper is to show that this is indeed the case.

The organization of the paper is as follows. In $\$ 2$, we collect all of the standard symbols and the terms used throughout the paper. Section 3 deals with the problem discussed above. Let $N$ be a factor of type $I I I$ whose smooth flow of weights is $\left(X_{N}, F^{N}\right)$. Fix any dominant weight $\phi$ on $N$. We show that each Borel 1-cocycle $c$ on the ergodic $\mathbb{R}$-space $X_{N}$ with values in a locally compact group $K$ yields a coaction $\beta_{c}^{\phi}$ of $K$ on $N$ which fixes the centralizer $N_{\phi}$. We also prove that a different choice of a dominant weight produces a conjugate coaction, and that a cohomologous 1-cocycle gives rise to a cocycle conjugate coaction. Therefore, the crossed product by the coaction $\beta_{c}^{\phi}$ and its dual action depend only on the cohomology class $[c]$ up to conjugacy. The crossed product is called the skew-product of $N$ by $c$. If $K$ equals $\mathbb{T}$, then our construction of $\beta_{c}^{\phi}$ amounts to the Connes-Takesaki extended modular automorphism group construction explained before. Hence, we call $\beta_{c}^{\phi}$ the extended modular coaction associated with $\phi$ and $c$. In $\S 4$, we characterize extended modular coactions as coactions that leave the centralizer of a dominant weight pointwise invariant. In $§ 5$, we discuss the Connes spectra of extended modular coactions. It is proven that the asymptotic range of $c$ is always contained in the Connes spectrum of $\beta_{c}^{\phi}$. Section 6 is concerned with crossed products by extended modular coactions and their smooth flows of weights. Thanks to the result in $\$ 5$, the extended modular coaction $\beta_{c}^{\phi}$ turns out to be strictly outer [36] if $c$ is a cocycle having dense range. For such a cocycle $c$, we will give a complete description of the smooth flow of weights on the crossed product by $\beta_{c}^{\phi}$ (Theorem 6.4). This generalizes the result obtained by Izumi in [22]. In $\S 7$, we examine the (Murray-von Neumann) algebraic type of crossed products by extended modular coactions in a certain situation. Section 8 contains a study of the dual actions of extended modular coactions associated with 1-cocycles having dense range. We characterize such an action as an integrable, minimal action whose Connes-Takesaki module is faithful and integrable. In $\S 9$, we give some remark on Galois correspondence for actions considered in $\S 8$. For an integrable, minimal action $\alpha$ of a locally compact group $K$ on a factor $M$ of type $I I I$ whose Connes-Takesaki module is faithful and integrable, we show, with some additional assumption, that an intermediate subfactor $L$ of the inclusion $M^{\alpha} \subseteq M$ is of the form $L=M^{\alpha_{H}}$ for a closed subgroup $H$ of $K$ if and only if there exist faithful normal semifinite operator-valued weights from $M$ to $L$ and from $L$ to $M^{\alpha}$. In Appendices A-D, we include some results which are used or referred to in the preceding sections. Usually, unitary 1-cocycles for group actions on von Neumann algebras are assumed to be $\sigma$-strongly* continuous with respect to the group parameters. It is, however, widely known (see [31, Notes 20.15]) that the $\sigma$-strong* continuity requirement can be replaced, without modifying the notion, by the measurability 
condition we adopt in this paper. This is folklore among specialists, but we could not locate literature that exhibits a concrete proof. So we include one here that fully utilizes the argument in [5]. Appendices A-D also contain another construction of the skew-product algebra obtained in $\S 6$. This is used in $\$ 9$ and seems to be of independent interest. Finally, we prove that, for any properly ergodic flow space $X$ and any amenable locally compact group $K$, there is a Borel 1-cocycle $c: \mathbb{R} \times X \rightarrow K$ having dense range.

\section{Notation and terminology}

Throughout this paper, we assume that all von Neumann algebras have separable preduals.

For a faithful normal semifinite weight $\phi$ on a von Neumann algebra $M$, we set

$$
\mathfrak{n}_{\phi}:=\left\{x \in M: \phi\left(x^{*} x\right)<\infty\right\}, \quad \mathfrak{m}_{\phi}:=\mathfrak{n}_{\phi}^{*} \mathfrak{n}_{\phi}, \quad \mathfrak{m}_{\phi}^{+}:=\mathfrak{m}_{\phi} \cap M_{+}
$$

More generally, for an operator-valued weight $T$ from a von Neumann algebra $M$ to a von Neumann subalgebra $N$, we set

$$
\mathfrak{n}_{T}:=\left\{x \in M: T\left(x^{*} x\right) \in N_{+}\right\}, \quad \mathfrak{m}_{T}:=\mathfrak{n}_{T}^{*} \mathfrak{n}_{T}, \quad \mathfrak{m}_{T}^{+}:=\mathfrak{m}_{T} \cap M_{+} .
$$

The Hilbert space obtained from $\phi$ by the Gelfand-Naimark-Segal (GNS)-construction will be denoted by $H_{\phi}$, and we let $\Lambda_{\phi}: \mathfrak{n}_{\phi} \rightarrow H_{\phi}$ stand for the natural injection. As usual, we use the symbols $J_{\phi}, \Delta_{\phi}$ to denote the modular conjugation and modular operator associated with $\phi$.

For a Hilbert space $H, B(H)$ stands for the algebra of all bounded operators on $H$.

In this paper, any locally compact group will be assumed to be second countable. Let $K$ be a locally compact group. Unless otherwise mentioned, we always consider a left Haar measure on $K$, which we denote by $m_{K}$. We denote by $W^{*}(K)$ the von Neumann algebra generated by the left regular representation $\lambda_{K}$ on $L^{2}(K)$. Remark that $W^{*}(K)$ is the left von Neumann algebra of the left Hilbert algebra $C_{c}(K)$ of all continuous functions on $K$ with compact support, where we consider on $C_{c}(K)$ the usual convolution and involution. The faithful semifinite normal weight on $W^{*}(K)$ associated with the left Hilbert algebra $C_{c}(K)$ is denoted by $\varphi_{K}$, the Plancherel weight on $W^{*}(K)$. It is known that there exists a unital normal injective $*$-homomorphism $\Delta_{K}$ from $W^{*}(K)$ into $W^{*}(K) \otimes W^{*}(K)$ characterized by the identity $\Delta_{K}\left(\lambda_{K}(k)\right)=\lambda_{K}(k) \otimes \lambda_{K}(k)$ for any $k \in K$. In fact, $\Delta_{K}$ is defined concretely by

$$
\Delta_{K}(x):=W_{K}^{*}(1 \otimes x) W_{K} \quad\left(x \in W^{*}(K)\right),
$$

where $W_{K}$ is the unitary given by $\left\{W_{K} \xi\right\}(k, h)=\xi(h k, h)\left(\xi \in L^{2}(K \times K)\right)$.

We denote the predual of $W^{*}(K)$ by $A(K)$, the Fourier algebra of $K$ (see [10]). Every element $\omega$ in $A(K)$ is regarded as a continuous function on $K$ given by $k \in K \mapsto$ $\omega\left(\lambda_{K}(k)^{*}\right)$

A coaction of $K$ on a von Neumann algebra $M$ is, by definition, a unital normal injective $*$-homomorphism $\alpha$ from $M$ into $W^{*}(K) \otimes M$ satisfying $\left(\Delta_{K} \otimes \mathrm{id}_{M}\right) \circ \alpha=$ $\left(\operatorname{id}_{W^{*}(K)} \otimes \alpha\right) \circ \alpha$.

Suppose that $\alpha$ is a coaction of $K$ on a von Neumann algebra $M$. 
(1) The fixed-point algebra of $\alpha$ is a von Neumann subalgebra $M^{\alpha}$ of $M$ defined by $M^{\alpha}:=\{a \in M: \alpha(a)=1 \otimes a\}$. We use the same notation for the fixed-point algebra of a group action.

(2) The map $T_{\alpha}$ defined by $T_{\alpha}(a):=\left(\varphi_{K} \otimes \mathrm{id}_{M}\right)(\alpha(a))$ is an operator-valued weight from $M$ to $M^{\alpha}$. The coaction $\alpha$ is said to be integrable if $T_{\alpha}$ is semifinite. (For an action $\beta$ of $K$ on $M$, the mapping $a \in M_{+} \mapsto \int_{K} \beta_{k}(a) d k$ defines a faithful normal operator-valued weight $T_{\beta}$ from $M$ to the fixed-point algebra $M^{\beta}$. We say that $\beta$ is integrable if $T_{\beta}$ is semifinite.)

(3) The crossed product of $M$ by $\alpha$ is the von Neumann algebra $\widehat{K}_{\alpha} \ltimes M:=(\alpha(M) \cup$ $\left.L^{\infty}(K) \otimes \mathbb{C}\right)^{\prime \prime}$. (In this paper, we adopt the notation in [35] for crossed products by (quantum) group actions. Hence, in the crossed product notation, the (quantum) groups appear on the left, while algebras appear on the right, such as $\widehat{K}_{\alpha} \ltimes M$, $\mathbb{R}_{\sigma^{\phi}} \ltimes M$, and so on.)

(4) For each $k \in K, \hat{\alpha}_{k}:=\left.\operatorname{Ad}\left(\rho_{K}(k) \otimes 1\right)\right|_{\widehat{K}_{\alpha} \ltimes M}$ defines a $*$-automorphism of $\widehat{K}_{\alpha} \ltimes M$, where $\rho_{K}$ is the right regular representation of $K$. We call $\hat{\alpha}$ the dual action of $\alpha$. (For the definition of the dual action of an action of a locally compact abelian group, see [31].)

(5) The map $T_{\hat{\alpha}}$ defined by $T_{\hat{\alpha}}(x):=\int_{K} \hat{\alpha}_{k}(x) d k$ is an operator-valued weight from $\widehat{K}_{\alpha} \ltimes M$ to $\alpha(M)$. For a normal weight $\psi$ on $M, \tilde{\psi}:=\psi \circ \alpha^{-1} \circ T_{\hat{\alpha}}$ is a normal weight on $\widehat{K}_{\alpha} \ltimes M$. It is called the dual weight of $\psi$.

(6) Define the coaction $\tilde{\alpha}$ of $K$ on $B\left(L^{2}(K)\right) \otimes M$ (the stabilization of $\alpha$ ) by

$$
\tilde{\alpha}:=\operatorname{Ad}\left(W_{K} \otimes 1\right) \circ(\sigma \otimes \mathrm{id}) \circ(\mathrm{id} \otimes \alpha) .
$$

It is known that $\left(B\left(L^{2}(K)\right) \otimes M\right)^{\tilde{\alpha}}=\widehat{K}_{\alpha} \ltimes M$.

(7) A unitary $V \in W^{*}(K) \otimes M$ is called an $\alpha$-1-cocycle if it satisfies $\left(\Delta_{K} \otimes \mathrm{id}\right)(V)=$ $V_{23}($ id $\otimes \alpha)(V)$. If $V$ is such a unitary, then $\operatorname{Ad} V \circ \alpha$ is again a coaction of $K$ on $M$. Let $\beta$ be another coaction of $K$ on $M$. We say that $\alpha$ is cocycle conjugate to $\beta$ if there exist a $*$-automorphism $\pi$ of $M$ and an $\alpha$-1-cocycle $V$ such that (id $\otimes \pi) \circ \beta \circ \pi^{-1}=\operatorname{Ad} V \circ \alpha$.

For the spectral theory for coactions such as the (Arveson) spectrum, the Connes spectrum and so on, we refer the reader to $[\mathbf{2 7}, \mathbf{2 8}]$.

Let $G, K$ be locally compact groups, and $X$ be a standard Borel $G$-space with quasiinvariant probability measure $\mu$. A Borel map $c: G \times X \rightarrow K$ is called a Borel 1 -cocycle if $c(g h, x)=c(g, h x) c(h, x)$ for any $g, h \in G$ and $x \in X$. Two Borel 1-cocycles $c, c^{\prime}: G \times X \rightarrow K$ are said to be cohomologous if there is a Borel map $q: X \rightarrow K$ such that, for each $g \in G, c^{\prime}(g, x)=q(g x)^{-1} c(g, x) q(x)$ for almost every $x \in X$.

Let $c: G \times X \rightarrow K$ be a Borel 1-cocycle as above. The essential range of $c$ is the smallest closed subset $\sigma(c)$ of $K$ such that $c^{-1}(\sigma(c))$ has complement of measure zero. The asymptotic range (or real image) $r^{*}(c)$ of $c$ (see $[\mathbf{1 3}, \mathbf{3 0}]$ ) is, by definition, $\bigcap\left\{\sigma\left(c_{B}\right): B \subseteq X, \mu(B)>0\right\}$, where $c_{B}$ stands for the restriction of $c$ to the subgroupoid $\{(g, x): g x, x \in B\}$. There is a natural $G$-action on the product standard Borel space $\left(K \times X, m_{K} \times \mu\right)$ defined by $g \cdot(k, x):=(c(g, x) k, g x)$. We call this action the skewproduct action associated with $c$, and often write $K \times{ }_{c} X$ in short for $\left(K \times X, m_{K} \times \mu\right)$ with this special action. If both the original $G$-action on $X$ and the skew-product action are ergodic, then we say that $c$ has dense range. 
Let $M$ be an infinite factor. Then one can associate in a functorial manner an ergodic abelian covariant system $\left(P_{M}, \mathbb{R}, \mathcal{F}^{M}\right)$, called the smooth flow of weights on $M$ (see [9]). One way to concretely realize this system is the following. Take a faithful normal semifinite weight $\phi$ on $M$. Then one obtains an abelian covariant system $\left(Z\left(\mathbb{R}_{\sigma \phi \ltimes} \ltimes\right), \mathbb{R}, \widehat{\sigma^{\phi}}\right)$, where $Z(N)$ in general stands for the center of a von Neumann algebra $N$ and $\widehat{\sigma^{\phi}}$ indicates the dual action of the modular automorphism group $\sigma^{\phi}$. It is well-known that this system is conjugate to the smooth flow of weights on $M$. Another equivalent realization by using a dominant weight [9] is given in the next section. In any case, there exist a (not necessarily unique) standard Borel probability space $\left(X_{M}, \mu_{M}\right)$ and an ergodic flow $\left\{F_{t}^{M}\right\}_{t \in \mathbb{R}}$ on $\left(X_{M}, \mu_{M}\right)$ such that $P_{M}=L^{\infty}\left(X_{M}, \mu_{M}\right)$ and $\mathcal{F}_{t}^{M}(f)=f \circ F_{-t}^{M}$ for $f \in L^{\infty}\left(X_{M}, \mu_{M}\right)$ and $t \in \mathbb{R}$. We also call $\left(X_{M}, F^{M}\right)$ the smooth flow of weights on $M$. The set of all Borel 1-cocycles $c: \mathbb{R} \times X_{M} \rightarrow K$ on $\left(X_{M}, F^{M}\right)$ will be denoted by $Z^{1}\left(F^{M}, K\right)$. The group of all non-singular Borel automorphisms on $X_{M}$ commuting with all $\left\{F_{t}^{M}\right\}$ will be denoted by $\operatorname{Aut}\left(F^{M}\right)$.

3. Coactions derived from 1-cocycles on the space of the smooth flow of weights: extended modular coactions

The goal of this section is to construct, from a Borel 1-cocycle on the smooth flow of weights on a type $I I I$ factor $N$ with a dominant weight $\phi$, a coaction on $N$ whose fixedpoint algebra contains the centralizer $N_{\phi}$ (Theorem 3.1).

Let $N$ be a factor of type $I I I$, and fix a dominant weight $\phi$ on $N$. We regard $N$ as represented standardly on the GNS Hilbert space $H_{\phi}$ obtained from $\phi$.

By the dominancy of $\phi$, we have a continuous decomposition of $N(\mathrm{cf} .[\mathbf{9}, \mathbf{3 1}, 33])$ as follows.

(1) The centralizer $N_{\phi}$ of $\phi$ is of type $I_{\infty}$.

(2) There exists a one-parameter unitary group $\{u(t)\}_{t \in \mathbb{R}}$ in $N$ satisfying $N=\left(N_{\phi} \cup\right.$ $\left.\{u(t)\}_{t \in \mathbb{R}}\right)^{\prime \prime}$ and $u(t) N_{\phi} u(t)=N_{\phi}$ for all $t \in \mathbb{R}$.

(3) If we set $\theta_{t}:=\left.\operatorname{Ad} u(t)\right|_{N_{\phi}} \in \operatorname{Aut}\left(N_{\phi}\right)(t \in \mathbb{R})$, then there is a $*$-isomorphism $\Psi$ from the crossed product $\mathbb{R}_{\theta} \ltimes N_{\phi}$ onto $N$ such that:

(i) $\Psi\left(\pi_{\theta}(x)\right)=x$ for all $x \in N_{\phi}$;

(ii) $\Psi\left(\lambda_{\mathbb{R}}(t) \otimes 1\right)=u(t)$ for all $t \in \mathbb{R}$;

(iii) the dual action $\hat{\theta}$ is conjugate to the modular automorphism group $\left\{\sigma^{\phi}\right\}$, i.e. $\Psi \circ \hat{\theta}_{t} \circ \Psi^{-1}=\sigma_{t}^{\phi}$ for any $t \in \mathbb{R}$.

(4) There exists a faithful normal semifinite trace $\tau$ on $N_{\phi}$ satisfying $\tau \circ \theta_{t}=e^{-t} \tau$ for all $t \in \mathbb{R}$.

The smooth flow of weights on $N$ is then the system $\left(Z\left(N_{\phi}\right), \mathbb{R}, \theta\right)$. So there exist a standard Borel space $X_{N}$, a probability measure $\mu$ on $X_{N}$ and an ergodic flow $F^{N}$ on $X_{N}$ such that $Z\left(N_{\phi}\right)=L^{\infty}\left(X_{N}, \mu\right)$ and $\theta_{t}(f)(x)=f\left(F_{-t}^{N} x\right)$ for $f \in L^{\infty}\left(X_{N}, \mu\right), t \in \mathbb{R}$ and $x \in X_{N}$. We often simply write $t x$ for $F_{t}^{N} x$.

We consider a central decomposition of $N_{\phi}$

$$
\left\{N_{\phi}, H_{\tau}\right\}=\int_{X_{N}}^{\oplus}\left\{N_{\phi}(x), H_{\tau}(x)\right\} d \mu(x)
$$

$\operatorname{over}\left(X_{N}, \mu\right)$. 
Now assume that we are given a Borel 1-cocycle $c \in Z^{1}\left(F^{N}, K\right)$ for a locally compact group $K$. Since the map $(t, x) \in \mathbb{R} \times X_{N} \longmapsto \lambda_{K}(c(t, x)) \in W^{*}(K)$ is a bounded Borel function, it determines an element $U_{c}$ in $L^{\infty}\left(\mathbb{R} \times X_{N}, W^{*}(K)\right)=$ $W^{*}(K) \otimes L^{\infty}(\mathbb{R}) \otimes Z\left(N_{\phi}\right)$. Clearly, $U_{c}$ is a unitary. It is also straightforward to check that $U_{c}$ satisfies

$$
\left(\Delta_{K} \otimes \operatorname{id}_{L^{\infty}(\mathbb{R})} \otimes \operatorname{id}_{Z\left(N_{\phi}\right)}\right)\left(U_{c}\right)=\left(U_{c}\right)_{234}\left(U_{c}\right)_{134}
$$

From this, it follows that the equation

$$
\beta_{0}^{\phi}(X):=U_{c}(1 \otimes X) U_{c}^{*} \quad\left(X \in B\left(L^{2}(\mathbb{R})\right) \otimes B\left(H_{\tau}\right)\right)
$$

defines a coaction $\beta_{0}^{\phi}$ of $K$ on $B\left(L^{2}(\mathbb{R})\right) \otimes B\left(H_{\tau}\right)$.

In the meantime, $L^{2}(K) \otimes L^{2}(\mathbb{R}) \otimes H_{\tau}$ admits a direct integral decomposition

$$
L^{2}(K) \otimes L^{2}(\mathbb{R}) \otimes H_{\tau}=\int_{X_{N}}^{\oplus} L^{2}(K) \otimes L^{2}(\mathbb{R}) \otimes H_{\tau}(x) d \mu(x) .
$$

Along this decomposition, $U_{c}$ is decomposed into $\int_{X_{N}}^{\oplus} U_{c}(x) d \mu(x)$, where, for any $x \in X_{N}, U_{c}(x)$ may be assumed to act as follows: $\left\{U_{c}(x) \eta\right\}(k, t)=\eta\left(c(t, x)^{-1} k, t\right)$ for $\eta \in L^{2}\left(K \times \mathbb{R}, H_{\tau}(x)\right)$.

THEOREM 3.1. The restriction of $\beta_{0}^{\phi}$ to the crossed product $\mathbb{R}_{\theta} \ltimes N_{\phi}$ determines a coaction of $K$ on $\mathbb{R}_{\theta} \ltimes N_{\phi}$. In fact, we have

$$
\begin{gathered}
\beta_{0}^{\phi}\left(\pi_{\theta}(a)\right)=1 \otimes \pi_{\theta}(a) \quad\left(a \in N_{\phi}\right), \\
\beta_{0}^{\phi}\left(\lambda_{\mathbb{R}}(s) \otimes 1\right)=\left(\operatorname{id}_{W^{*}(K)} \otimes \pi_{\theta}\right)\left(Q_{s}\right)\left(1 \otimes \lambda_{\mathbb{R}}(s) \otimes 1\right) \quad(s \in \mathbb{R}),
\end{gathered}
$$

where $Q_{S}$ is a unitary in $W^{*}(K) \otimes Z\left(N_{\phi}\right)=L^{\infty}\left(X_{N}, W^{*}(K)\right)$ defined by the Borel function: $x \in X_{N} \mapsto \lambda_{K}(c(-s, x))^{*} \in W^{*}(K)$. Moreover, a different choice of a dominant weight on $N$ gives rise to a conjugate coaction in this procedure.

Proof. Since $\pi_{\theta}\left(N_{\phi}\right)$ is contained in $L^{\infty}(\mathbb{R}) \otimes N_{\phi}, U_{c}$ commutes with $1 \otimes \pi_{\theta}(a)$ for all $a \in N_{\phi}$. Thus, (3.2) follows.

To show (3.3), we first note that $U_{c}\left(1 \otimes \lambda_{\mathbb{R}}(s) \otimes 1\right) U_{c}^{*}$ acts in the following manner:

$$
\left\{U_{c}\left(1 \otimes \lambda_{\mathbb{R}}(s) \otimes 1\right) U_{c}^{*} \xi\right\}_{x}(k, t)=\xi_{x}(c(-s, t x) k, t-s),
$$

where

$$
\xi=\int_{X_{N}}^{\oplus} \xi_{x} d \mu(x) \in L^{2}(K) \otimes L^{2}(\mathbb{R}) \otimes H_{\tau}=\int_{X_{N}}^{\oplus} L^{2}\left(K \times \mathbb{R}, H_{\tau}(x)\right) d \mu(x) .
$$

This may be verified by a direct calculation using the cocycle property of $c$. If $Q_{s}$ is the unitary defined in the statement of this theorem, then, by (3.4), we get

$$
\begin{aligned}
\left\{U_{c}\left(1 \otimes \lambda_{\mathbb{R}}(s) \otimes 1\right) U_{c}^{*} \xi\right\}_{x} & =\left\{\left(1 \otimes \lambda_{\mathbb{R}}(s) \otimes 1\right) \xi\right\}_{x}(c(-s, t x) k, t) \\
& =\left\{\left(\operatorname{id}_{W^{*}(K)} \otimes \pi_{\theta}\right)\left(Q_{s}\right)\left(1 \otimes \lambda_{\mathbb{R}}(s) \otimes 1\right) \xi\right\}_{x}(k, t) .
\end{aligned}
$$

Thus (3.3) is verified. From (3.2) and (3.3), we have $\beta_{0}^{\phi}\left(\mathbb{R}_{\theta} \ltimes N_{\phi}\right) \subseteq W^{*}(K) \otimes \mathbb{R}_{\theta} \ltimes N_{\phi}$. It follows that the restriction of $\beta_{0}^{\phi}$ to $\mathbb{R}_{\theta} \ltimes N_{\phi}$ defines a coaction of $K$ on it. 
Finally, let $\omega$ be another dominant weight on $N$. By [9, Ch. II, Theorem 1.1], there is a unitary $u \in N$ such that $\omega(a)=\phi\left(u a u^{*}\right)$ for $a \in N_{+}$. Then it is easy to check with the aid of (3.2) and (3.3) that one has $\beta_{0}^{\phi} \circ(\mathrm{id} \otimes \operatorname{Ad} u)=(\mathrm{id} \otimes \mathrm{id} \otimes \operatorname{Ad} u) \circ \beta_{0}^{\omega}$. Therefore, $\beta_{0}^{\omega}$ is conjugate to $\beta_{0}^{\phi}$.

Definition 3.2. Thanks to Theorem 3.1, the map $\left.\left(\operatorname{id}_{W^{*}(K)} \otimes \Psi\right) \circ \beta_{0}^{\phi}\right|_{\mathbb{R}_{\theta} \ltimes N_{\phi}} \circ \Psi^{-1}$ is a coaction of $K$ on $N$. We denote it by $\beta_{c}^{\phi}$, and call it the extended modular coaction associated with $\phi$ and $c$. From Theorem 3.1, we have

$$
\begin{gathered}
\beta_{c}^{\phi}(a)=1 \otimes a \quad\left(a \in N_{\phi}\right), \\
\beta_{c}^{\phi}(u(s))=Q_{s}(1 \otimes u(s)) \quad(s \in \mathbb{R}) .
\end{gathered}
$$

Proposition 3.3. Let $c, c^{\prime}: \mathbb{R} \times X_{N} \rightarrow K$ be Borel 1-cocycles. They are cohomologous to each other if and only if there exists a $\beta_{c}^{\phi}$-1-cocycle $R$ such that $\beta_{c^{\prime}}^{\phi}=\operatorname{Ad} R \circ \beta_{c}^{\phi}$ (in particular, the coactions are cocycle conjugate).

Proof. Suppose that $c$ and $c^{\prime}$ are cohomologous. So there is a Borel map $q: X \rightarrow K$ such that, for each $t \in \mathbb{R}, c^{\prime}(t, x)=q\left(F_{t}^{N} x\right)^{-1} c(t, x) q(x)$ for almost every $x \in X_{N}$. Consider the Borel map $V_{q}: x \in X_{N} \mapsto \lambda_{K}(q(x))^{*} \in W^{*}(K)$. Then $V_{q}$ is a unitary in $L^{\infty}\left(X_{N}, W^{*}(K)\right)=W^{*}(K) \otimes Z\left(N_{\phi}\right)$. Since $\Delta_{K}\left(V_{q}(x)\right)=V_{q}(x) \otimes V_{q}(x)$, we obtain $\left(\Delta_{K} \otimes \mathrm{id}\right)\left(V_{q}\right)=\left(V_{q}\right)_{23}\left(V_{q}\right)_{13}$. By (3.5), we have (id $\left.\otimes \beta_{c}^{\phi}\right)\left(V_{q}\right)=\left(V_{q}\right)_{13}$. This means that $V_{q}$ is a $\beta_{c}^{\phi}$-1-cocycle. Set $\beta:=\operatorname{Ad} V_{q} \circ \beta_{c}^{\phi}$. We have $\beta(a)=1 \otimes a$ for any $a \in N_{\phi}$. Let $\{u(t)\}$ and $\theta_{t}=\operatorname{Ad} u(t)$ be as in the beginning of this section. Fix any $s \in \mathbb{R}$. Then $\beta(u(s))=V_{q} Q_{s}^{c}(1 \otimes u(s)) V_{q}^{*}=V_{q} Q_{s}^{c}\left(\mathrm{id} \otimes \theta_{s}\right)\left(V_{q}^{*}\right)(1 \otimes u(s))$, where $Q_{s}^{c}$ is the unitary in Theorem 3.1 constructed from $c$. Note that the unitary $V_{q} Q_{s}^{c}$ (id $\left.\otimes \theta_{s}\right)\left(V_{q}^{*}\right)$ is regarded as a $W^{*}(K)$-valued Borel function on $X_{N}$ given by

$$
x \in X_{N} \longmapsto \lambda_{K}\left(q(x)^{-1}\right) \lambda_{K}\left(c(-s, x)^{-1}\right) \lambda_{K}\left(q\left(F_{-s}^{N} x\right)\right)=\lambda_{K}\left(c^{\prime}(-s, x)^{-1}\right) .
$$

This shows $V_{q} Q_{s}^{c}\left(\mathrm{id} \otimes \theta_{s}\right)\left(V_{q}^{*}\right)=Q_{s}^{c^{\prime}}$, where $Q_{s}^{c^{\prime}}$ is, of course, the unitary in Theorem 3.1 constructed from $c^{\prime}$. So we obtain $\beta(u(s))=Q_{s}^{c^{\prime}}(1 \otimes u(s))$. Therefore, $\beta=\beta_{c^{\prime}}^{\phi}$. Hence, we may take $V_{q}$ for the desired unitary $R$.

Suppose next that there exists a $\beta_{c}^{\phi}$-1-cocycle $R$ such that $\beta_{c^{\prime}}^{\phi}=\operatorname{Ad} R \circ \beta_{c}^{\phi}$. From (3.5) and the relative commutant theorem, we find that $R$ belongs to $W^{*}(K) \otimes Z\left(N_{\phi}\right)$. Thus, $R$ can be viewed as a $W^{*}(K)$-valued Borel function on $X_{N}$. Since $\left(\Delta_{K} \otimes \mathrm{id}\right)(R)=$ $R_{23}\left(\mathrm{id} \otimes \beta_{c}^{\phi}\right)(R)=R_{23} R_{13}$, it follows that $\Delta_{K}(R(x))=R(x) \otimes R(x)$. Hence, we may and do assume that there is a Borel function $p: X_{N} \rightarrow K$ such that $R(x)=\lambda_{K}(p(x))^{*}$. By (3.6), we get $Q_{s}^{c^{\prime}}=R Q_{s}^{c}(1 \otimes u(s)) R\left(1 \otimes u(s)^{*}\right)=R Q_{s}^{c}\left(\mathrm{id} \otimes \theta_{s}\right)\left(R^{*}\right)$ for any $s \in \mathbb{R}$. As in previous paragraph, this identity means that, for almost every $x \in X_{N}$, we have

$$
c(-s, x)^{-1}=p(x)^{-1} c(-s, x) p\left(F_{-s}^{N} x\right) .
$$

Therefore, $c^{\prime}$ is cohomologous to $c$.

As cocycle conjugate covariant systems produce conjugate dual covariant systems, the next corollary immediately follows from Proposition 3.3. 


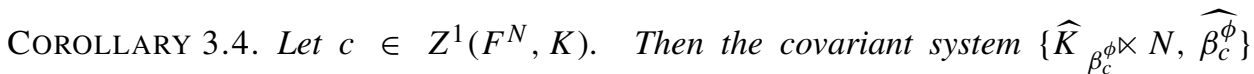
depends only on the cohomology class [c], up to conjugacy. As in [22], we often write $L^{\infty}(K) \otimes_{c} N$ for the crossed product $\widehat{K}_{\beta_{c}^{\phi}} \ltimes N$, and call it the skew-product (algebra) of $N$ byc.

Corollary 3.5. Let $c \in Z^{1}\left(F^{N}, K\right)$ and $\phi$ be a dominant weight on $N$ as before. Then $c$ is cohomologous to the trivial cocycle if and only if $\beta_{c}^{\phi}$ is inner in the sense that there exists a unitary $V \in W^{*}(K) \otimes N$ satisfying $\left(\Delta_{K} \otimes \mathrm{id}\right)(V)=V_{23} V_{13}$ and $\beta_{c}^{\phi}(a)=V(1 \otimes a) V^{*}$ for all $a \in N$.

Proof. This follows at once from Proposition 3.3.

Proposition 3.6. Let $c: \mathbb{R} \times X_{N} \rightarrow K$ be a Borel 1-cocycle. Suppose that $K$ is abelian. It is well known that the coaction $\beta_{c}^{\phi}$ of $K$ corresponds to an automorphic action, still denoted by $\beta_{c}^{\phi}$, of the dual group $\hat{K}$ on $N$. For any $\gamma \in \hat{K}$ and any $t \in \mathbb{R}$, define $a$ Borel function $\hat{c}_{\gamma}(t)$ in $L^{\infty}\left(X_{N}\right)$ by $\hat{c}_{\gamma}(t)(x):=\langle c(-t, x), \gamma\rangle$, where $\langle\cdot, \cdot\rangle: K \times \hat{K} \rightarrow \mathbb{T}$ is the natural pairing.

(1) For each $\gamma \in \hat{K}, \hat{c}_{\gamma}$ belongs to $Z_{\mathcal{F}^{N}}^{1}\left(\mathbb{R}, \mathcal{U}\left(P_{N}\right)\right)$, that is, $\hat{c}_{\gamma}$ is a $\mathcal{F}^{N}$-1-cocycle in the sense of [9]. (See Appendix A for the symbol $Z_{\mathcal{F}^{N}}^{1}\left(\mathbb{R}, \mathcal{U}\left(P_{N}\right)\right)$ ).)

(2) Let $\sigma_{\hat{c}_{\gamma}}^{\phi}$ be the extended modular automorphism of $N$ associated with $\mathcal{F}^{N}$-cocycle $\hat{c}_{\gamma}$ (cf. [9, §IV.2]). Then the action $\gamma \in \hat{K} \mapsto \sigma_{\hat{c}_{\gamma}}^{\phi} \in \operatorname{Aut}(N)$ of $\hat{K}$ on $N$ is exactly the action $\beta_{c}^{\phi}$.

Proof. Part (1) follows from the results of Appendix A.

For Part (2), let $\mathcal{F}: L^{2}(K) \rightarrow L^{2}(\hat{K})$ be the Fourier transform. Thus, it satisfies

$$
\{\mathcal{F} g\}(\gamma)=\int_{K} \overline{\langle k, \gamma\rangle} g(k) d k
$$

for any compactly supported continuous function $g$ on $K$. Since $\mathcal{F} \lambda_{K}(k) \mathcal{F}^{*}$ is equal to the multiplication by the function $\overline{\langle k, \cdot\rangle}$ on $\hat{K}$, it follows from (3.2) and (3.3) that the automorphic action $\beta_{c}^{\phi}$ satisfies $\left(\beta_{c}^{\phi}\right)_{\gamma}(a)=a$ and $\left(\beta_{c}^{\phi}\right)_{\gamma}(u(s))=\hat{c}_{\gamma}(s) u(s)$ for all $a \in N_{\phi}, s \in \mathbb{R}$ and $\gamma \in \hat{K}$. This shows that, for each $\gamma \in \hat{K},\left(\beta_{c}^{\phi}\right)_{\gamma}$ is the extended modular automorphism associated with $\hat{c}_{\gamma}$.

This proposition would justify our terminology 'extended modular coaction' for $\beta_{c}^{\phi}$.

LEMMA 3.7. We have $\left(\operatorname{id}_{W^{*}(K)} \otimes \sigma_{t}^{\phi}\right) \circ \beta_{c}^{\phi}=\beta_{c}^{\phi} \circ \sigma_{t}^{\phi}$ for all $t \in \mathbb{R}$.

Proof. The claimed identity easily follows from (3.5), (3.6) and $\sigma_{t}^{\phi}(u(s))=e^{-i s t} u(s)$.

PROPOSITION 3.8. The weight $\phi$ is 1-invariant with respect to $\beta_{c}^{\phi}$ in the sense of [35], that is, it satisfies

$$
\phi\left(\left(\omega_{\xi} \otimes \operatorname{id}_{N}\right)\left(\beta_{c}^{\phi}(a)\right)\right)=\|\xi\|^{2} \phi(a)
$$

for any $\xi \in L^{2}(K)$ and $a \in \mathfrak{m}_{\phi}^{+}$.

Proof. We know that the equation

$$
T_{\phi}(a):=\int_{\mathbb{R}} \sigma_{t}^{\phi}(a) d t \quad\left(a \in N_{+}\right)
$$


defines a faithful normal semifinite operator-valued weight from $N$ to $N_{\phi}$ that satisfies $\phi=\tau \circ T_{\phi}$. From this and Lemma 3.7, we have, for any $a \in N_{+}$,

$$
\begin{aligned}
\left(\operatorname{id}_{W^{*}(K)} \otimes T_{\phi}\right)\left(\beta_{c}^{\phi}(a)\right) & =\int_{\mathbb{R}}\left(\operatorname{id}_{W^{*}(K)} \otimes \sigma_{t}^{\phi}\right)\left(\beta_{c}^{\phi}(a)\right) d t \\
& =\int_{\mathbb{R}} \beta_{c}^{\phi}\left(\sigma_{t}^{\phi}(a)\right) d t=1 \otimes T_{\phi}(a) .
\end{aligned}
$$

By this result, if $a \in \mathfrak{m}_{\phi}^{+}$, we get

$$
\begin{aligned}
\phi\left(\left(\omega_{\xi} \otimes \mathrm{id}_{N}\right)\left(\beta_{c}^{\phi}(a)\right)\right) & =\left(\omega_{\xi} \otimes \phi\right)\left(\beta_{c}^{\phi}(a)\right)=\left(\omega_{\xi} \otimes \tau \circ T_{\phi}\right)\left(\beta_{c}^{\phi}(a)\right) \\
& =\left(\omega_{\xi} \otimes \tau\right)\left(\left(\operatorname{id}_{W^{*}(K)} \otimes T_{\phi}\right)\left(\beta_{c}^{\phi}(a)\right)\right) \\
& =\left(\omega_{\xi} \otimes \tau\right)\left(1 \otimes T_{\phi}(a)\right)=\|\xi\|^{2} \phi(a) .
\end{aligned}
$$

This completes the proof.

Remark. We close this section with a brief remark on the construction of the skewproduct $L^{\infty}(K) \otimes_{c} N$. In this section, the algebra $L^{\infty}(K) \otimes_{c} N$, or rather the covariant system $\left(L^{\infty}(K) \otimes_{c} N, K, \widehat{\beta_{c}^{\phi}}\right)$, was obtained by way of constructing the extended modular coaction $\beta_{c}^{\phi}$. Although we believe that our approach (by using extended modular coactions) is very important in its own right, we emphasize that there is another way of defining the skew-product algebra without introducing the notion of an extended modular coaction. This approach is fully illustrated in Appendix C. The idea is to directly construct a 'continuous decomposition' of the skew-product algebra from $N$ and $c$. We will make use of this approach in $\S 9$.

\section{Characterization of extended modular coactions}

The main result of this section (Theorem 4.1) asserts that extended modular coactions are exactly the coactions whose fixed-point algebras contain the centralizer of some dominant weight (the dominant weight may vary depending on a coaction). This, together with the results in the preceding section, would imply that the extended modular coaction is a right generalization of Connes-Takesaki's extended modular automorphism group.

As in the previous section, let $N$ be a factor of type $I I I$ and $\phi$ a dominant weight on $N$. We retain the notation introduced in the preceding section.

THEOREM 4.1. If a coaction $\beta$ of a locally compact group $K$ on $N$ satisfies $N_{\phi} \subseteq N^{\beta}$, then there exists a Borel 1-cocycle $c: \mathbb{R} \times X_{N} \rightarrow K$ such that $\beta=\beta_{c}^{\phi}$.

Proof. For any $s \in \mathbb{R}$, set $w(s):=\left(1 \otimes u(s)^{*}\right) \beta(u(s)) \in W^{*}(K) \otimes N$. For any $x \in N_{\phi}$, we have

$$
\begin{aligned}
(1 \otimes x) w(s) & =\left(1 \otimes u(s)^{*}\right)\left(1 \otimes \theta_{s}(x)\right) \beta(u(s))=\left(1 \otimes u(s)^{*}\right) \beta\left(\theta_{s}(x) u(s)\right) \\
& =\left(1 \otimes u(s)^{*}\right) \beta(u(s) x)=w(s)(1 \otimes x)
\end{aligned}
$$

Hence, by the relative commutant theorem [9, Ch. II, Theorem 5.1], we get

$$
w(s) \in W^{*}(K) \otimes N \cap\left(\mathbb{C} \otimes N_{\phi}\right)^{\prime}=W^{*}(K) \otimes N \cap\left(N_{\phi}\right)^{\prime}=W^{*}(K) \otimes Z\left(N_{\phi}\right) .
$$


Hence, $w(\cdot)$ belongs to $W^{*}(K) \otimes L^{\infty}(\mathbb{R}) \otimes Z\left(N_{\phi}\right)$, and thus can be regarded as a bounded Borel function from $\mathbb{R} \times X_{N}$ into $W^{*}(K)$.

In the meantime, we have

$$
\begin{aligned}
\left(\Delta_{K} \otimes \operatorname{id}_{N}\right)(w(s)) & =\left(1 \otimes 1 \otimes u(s)^{*}\right)\left(\Delta_{K} \otimes \operatorname{id}_{N}\right)(\beta(u(s))) \\
& =\left(1 \otimes 1 \otimes u(s)^{*}\right)\left(\operatorname{id}_{W^{*}(K)} \otimes \beta\right)(\beta(u(s))) \\
& =(1 \otimes w(s))\left(1 \otimes \beta\left(u(s)^{*}\right)\right)\left(\operatorname{id}_{W^{*}(K)} \otimes \beta\right)(\beta(u(s))) \\
& =w(s)_{23}\left(\operatorname{id}_{W^{*}(K)} \otimes \beta\right)(w(s)) \\
& =w(s)_{23} w(s)_{13} .
\end{aligned}
$$

From this, it follows that $w(s, x)$ satisfies $\Delta_{K}(w(s, x))=w(s, x) \otimes w(s, x)$ for any $s \in \mathbb{R}$ and $x \in X_{N}$. To sum up, there is a Borel map $c^{\prime}: \mathbb{R} \times X_{N} \rightarrow K$ such that $w(s, x)=\lambda_{K}\left(c^{\prime}(s, x)\right) \in W^{*}(K)$.

For $s, t \in \mathbb{R}$, we have

$$
\begin{aligned}
w(s+t) & =\left(1 \otimes u(s+t)^{*}\right) \beta(u(s+t))=\left(1 \otimes u(s+t)^{*}\right) \beta(u(s))(1 \otimes u(t)) w(t) \\
& =\left(1 \otimes u(t)^{*}\right) w(s)(1 \otimes u(t)) w(t)=\left(\operatorname{id}_{W^{*}(K)} \otimes \theta_{-t}\right)(w(s)) w(t) .
\end{aligned}
$$

This shows that, for all $s, t \in \mathbb{R}$, the map $c^{\prime}$ satisfies $c^{\prime}(s+t, x)=c^{\prime}(s, t x) c^{\prime}(t, x)$ for almost every $x \in X_{N}$. By [42, Theorem B.9], there exists a Borel 1-cocycle $c: \mathbb{R} \times X_{N} \rightarrow$ $K$ such that, for all $s \in \mathbb{R}, c(s, x)=c^{\prime}(s, x)$ for almost every $x \in X_{N}$. So we may assume from the outset that $w$ is determined by this cocycle $c$.

By definition, we have $\beta(u(s))=\left(\operatorname{id}_{W^{*}(K)} \otimes \theta_{s}\right)(w(s))(1 \otimes u(s))$. Note that, for each $s \in \mathbb{R}$, the unitary $\left(\operatorname{id}_{W^{*}(K)} \otimes \theta_{s}\right)(w(s))$ is determined by the function $x \in X_{N} \mapsto$ $\lambda(c(s,-s x)) \in W^{*}(K)$. By the cocycle property of $c$, we have $c(s,-s x)=c(-s, x)^{-1}$. Hence, $\left(\operatorname{id}_{W^{*}(K)} \otimes \theta_{s}\right)(w(s))$ is nothing but the unitary $Q_{s}$ in Theorem 3.1. Thus, we get $\beta(u(s))=Q_{s}(1 \otimes u(s))$ for any $s \in \mathbb{R}$. Therefore, we conclude that $\beta=\beta_{c}^{\phi}$.

Remark. The proof of the preceding theorem allows us to obtain the following claim: if a locally compact quantum group $\mathbb{G}$ (in the sense of Kustermans and Vaes [25]) admits a faithful action $\beta$ on a type $I I I$ factor $N$ for which there exists a dominant weight $\phi$ on $N$ satisfying $N_{\phi} \subseteq N^{\beta}$ (the fixed-point algebra of $\beta$ ), then $\mathbb{G}$ must be cocommutative. To verify this claim, let $\beta$ be such an action of the locally compact quantum group $\mathbb{G}=(M, \Delta, \varphi, \psi)$. We freely employ the notation used in [39] for locally compact quantum groups and their actions. Note that the proof of Theorem 4.1 is valid up to the second paragraph, except that we have $\Delta(w(s, x))=w(s, x) \otimes w(s, x)$ instead in the end. This means that, almost everywhere, $w(s, x)$ belongs to the so-called intrinsic group $I G(\mathbb{G})$ of $\mathbb{G}$, where $I G(\mathbb{G}):=\{u \in M: u$ is a unitary, $\Delta(u)=u \otimes u\}$. Hence, it follows that $w(s)$ belongs to $I G(\mathbb{G})^{\prime \prime} \otimes Z\left(N_{\phi}\right)$. In particular, $\beta(u(s))$ is in $I G(\mathbb{G})^{\prime \prime} \otimes N$ for all $s \in \mathbb{R}$, since $N=\left(N_{\phi} \cup\{u(s): s \in \mathbb{R}\}\right)^{\prime \prime}$. Owing to $N_{\phi} \subseteq N^{\beta}$, we have $\beta(N) \subseteq I G(\mathbb{G})^{\prime \prime} \otimes N$. From this and the assumption that $\beta$ is faithful, i.e. $\left\{(\operatorname{id} \otimes \omega)(\beta(a)): a \in N, \omega \in N_{*}\right\}^{\prime \prime}=M$, we find that $M$ is equal to $I G(\mathbb{G})^{\prime \prime}$. Therefore, $\mathbb{G}$ is cocommutative. Namely, there exists a unique locally compact group $K$ such that $M=W^{*}(K)$ and $\Delta=\Delta_{K}$. In particular, $\beta$ is a (faithful) coaction of this group $K$ on $N$ as in Theorem 4.1. 
Proposition 4.2. Let $c_{1}, c_{2} \in Z^{1}\left(F^{N}, K\right)$. If there exist $a *$-automorphism $\gamma$ of $N$ and $a \beta_{c_{1}}^{\phi}$-1-cocycle $V$ such that $\phi \circ \gamma=\phi$ and $(\mathrm{id} \otimes \gamma) \circ \beta_{c_{2}}^{\phi} \circ \gamma^{-1}=\operatorname{Ad} V \circ \beta_{c_{1}}^{\phi}$, then there exists a transformation $T \in \operatorname{Aut}\left(F^{N}\right)$ such that $c_{1}$ is cohomologous to $c_{2} \circ T^{-1}$, where $c_{2} \circ T^{-1}$ is a Borel 1-cocycle in $Z^{1}\left(F^{N}, K\right)$ given by $c_{2} \circ T^{-1}(s, x):=c_{2}\left(s, T^{-1} x\right)$.

Proof. Let $\gamma \in \operatorname{Aut}(N)$ and $V$ be as above. Since $\gamma\left(N_{\phi}\right)=N_{\phi}$, the coaction $\beta:=$ (id $\otimes \gamma$ ) $\circ \beta_{c_{2}}^{\phi} \circ \gamma^{-1}$ of $K$ on $N$ satisfies $N^{\alpha} \supseteq N_{\phi}$. By Theorem 4.1, $\beta$ is of the form $\beta=\beta_{c}^{\phi}$ for some $c \in Z^{1}\left(F^{N}, K\right)$. Then, by Proposition 3.3, $c$ is cohomologous to $c_{1}$. Let $v(s):=\gamma(u(s)) u(s)^{*} \in N_{\phi}$. It is easy to check that $v$ is a $\theta-1$-cocycle. By [9, Theorem 5.1], there exists a unitary $v \in N_{\phi}$ such that $v(s)=v^{*} \theta_{s}(v)$ for all $s \in \mathbb{R}$. From this and (3.6), we have

$$
\begin{aligned}
Q_{s}^{c}(1 \otimes u(s)) & =\beta_{c}^{\phi}(u(s))=(\mathrm{id} \otimes \gamma) \circ \beta_{c_{2}}^{\phi}\left(\gamma^{-1}(v) u(s) \gamma^{-1}\left(v^{*}\right)\right) \\
& =(\mathrm{id} \otimes \gamma)\left(\left(1 \otimes \gamma^{-1}(v)\right) Q_{s}^{c_{2}}(1 \otimes u(s))\left(1 \otimes \gamma^{-1}\left(v^{*}\right)\right)\right) \\
& =(1 \otimes v)(\mathrm{id} \otimes \gamma)\left(Q_{s}^{c_{2}}\right)(1 \otimes \gamma(u(s)))\left(1 \otimes v^{*}\right) \\
& =(1 \otimes v)(\mathrm{id} \otimes \gamma)\left(Q_{s}^{c_{2}}\right)\left(1 \otimes v^{*}\right)(1 \otimes u(s)) \\
& =(\mathrm{id} \otimes \gamma)\left(Q_{s}^{c_{2}}\right)(1 \otimes u(s)),
\end{aligned}
$$

where $Q^{c}$ and $Q_{s}^{c_{2}}$ are respectively the unitaries in Theorem 3.1 constructed from cocycles $c$ and $c_{2}$. From the above calculation, we obtain $Q_{s}^{c}=(\operatorname{id} \otimes \gamma)\left(Q_{s}^{c_{2}}\right)$ for any $s \in \mathbb{R}$. Let $T$ be the non-singular transformation on $X_{N}$ determined by the automorphism $\gamma \mid Z\left(N_{\phi}\right)$. Then we have $c(s, x)=c_{2}\left(s, T^{-1} x\right)$. If $z \in Z\left(N_{\phi}\right)$, then

$$
\begin{aligned}
\gamma \circ \theta_{s}(z) & =\gamma\left(u(s) z u(s)^{*}\right)=\gamma(u(s)) \gamma(z) \gamma\left(u(s)^{*}\right) \\
& =v u(s) v^{*} \gamma(z) v^{*} u(s)^{*} v=u(s) \gamma(z) u(s)^{*} \\
& =\theta_{s} \circ \gamma(z) .
\end{aligned}
$$

This shows that $T$ belongs to $\operatorname{Aut}\left(F^{N}\right)$.

5. The Connes spectrum of $\beta_{c}^{\phi}$

This section is concerned with a close link between the asymptotic range of $c \in$ $Z^{1}\left(F^{N}, K\right)$ and the Connes spectrum of the associated extended modular coaction.

Let $N$ be a factor of type $I I I$ and $\phi$ a dominant weight on $N$. We still employ the notation used in $\S 3$. With a locally compact group $K$, we fix a Borel 1-cocycle $c: \mathbb{R} \times X_{N} \rightarrow K$ in this section. We simply write $\beta$ for the coaction $\beta_{c}^{\phi}$.

THEOREM 5.1. The asymptotic range $r^{*}(c)$ of $c$ is contained in the Connes spectrum $\Gamma(\beta)$ of $\beta$.

Proof. We know that $N_{\phi}$ is contained in $N^{\beta}$. From this and the relative commutant theorem, it follows that $Z\left(N^{\beta}\right) \subseteq\left(N_{\phi}\right)^{\prime} \cap N=Z\left(N_{\phi}\right) \subseteq N^{\beta}$. Hence, we get

$$
\Gamma(\beta)=\bigcap\left\{\operatorname{Sp}\left(\beta^{e}\right): e \text { is non-zero projection in } Z\left(N_{\phi}\right)\right\},
$$

where $\beta^{e}$ is the restriction of $\beta$ to $e N e$.

Let $m$ denote the Lebesgue measure on $\mathbb{R}$. 
Suppose that $k$ belongs to the essential range $\sigma(c)$. Take any compact neighborhood $V$ of $k$. So $(m \times \mu)\left(c^{-1}(V)\right)>0$. For any $t \in \mathbb{R}$, put $B_{t}:=\left\{x \in X_{N}:(t, x) \in c^{-1}(V)\right\}$. Then

$$
\int_{\mathbb{R}} \mu\left(B_{t}\right) d t=(m \times \mu)\left(c^{-1}(V)\right)>0 .
$$

Choose an $s \in \mathbb{R}$ such that $\mu\left(B_{s}\right)>0$. Set $a:=\chi_{B_{s}} u(-s) \in N \backslash\{0\}$. Then, for any $\omega \in A(K)$ that vanishes on some neighborhood of $V$, we have

$\left(\omega \otimes \operatorname{id}_{N}\right)(\beta(a))=\left(\omega \otimes \operatorname{id}_{N}\right)\left(\left(1 \otimes \chi_{B_{s}}\right) Q_{-s}(1 \otimes u(-s))\right)=\chi_{B_{s}} \omega(c(s, \cdot)) u(-s)=0$,

because $\{s\} \times B_{s} \subseteq c^{-1}(V)$. From [28, Ch. IV, Lemma 1.2 (ii)], it follows that $\operatorname{Sp}_{\beta}(a)$ is contained in $V$. Hence, $a$ belongs to $N^{\beta}(V)$. By [28, Ch. IV, Lemma 1.2(iv)], $k$ is in $\operatorname{Sp}(\beta)$. Therefore, we conclude that $\sigma(c) \subseteq \operatorname{Sp}(\beta)$.

Take a non-zero projection $e$ in $Z\left(N_{\phi}\right)=L^{\infty}\left(X_{N}\right)$. So there is a Borel subset $B$ of $X_{N}$ such that $e=\chi_{B}$. Consider the reduction $\mathcal{G}_{B}$ of the measured groupoid $\mathcal{G}:=\mathbb{R} \times X_{N}$ to $B$ and the restriction $c_{B}:=\left.c\right|_{\mathcal{G}_{B}}$. We claim that the essential range $\sigma\left(c_{B}\right)$ of the 1-cocycle $c_{B}$ is contained in $\operatorname{Sp}\left(\beta^{e}\right)$. This can be verified as in the preceding paragraph. Indeed, suppose that $k$ is in $\sigma\left(c_{B}\right)$. For any compact neighborhood of $k$, we can choose an $s \in \mathbb{R}$ so that the $\mathbb{R}$-section $B_{s}$ of $\left(c_{B}\right)^{-1}(V)$ at $s$ has positive measure. Put $b:=\chi_{B_{s}} u(-s) e \in e N e \backslash\{0\}$. Then, for any $\omega \in A(K)$ that vanishes on some neighborhood of $V$, we have $\left(\omega \otimes \operatorname{id}_{e N e}\right)\left(\beta^{e}(b)\right)=0$. Hence, $b$ belongs to $(e N e)^{\beta^{e}}(V)$. This implies that $k$ is in $\operatorname{Sp}\left(\beta^{e}\right)$.

By the results of the previous paragraphs, we obtain

$$
\begin{aligned}
r^{*}(c) & =\bigcap\left\{\sigma\left(c_{B}\right): B \subseteq X_{N}, \mu(B)>0\right\} \\
& \subseteq \bigcap\left\{\operatorname{Sp}\left(\beta^{e}\right): e \text { is non-zero projection in } Z\left(N_{\phi}\right)\right\}=\Gamma(\beta) .
\end{aligned}
$$

This completes the proof.

It is interesting to study under what condition $r^{*}(c)$ coincides with $\Gamma\left(\beta_{c}^{\phi}\right)$, except the case of $r^{*}(c)$ being equal to $K$.

\section{The crossed product $\hat{K}_{\beta_{c}^{\phi}} \ltimes N$ and its smooth flow of weights}

This section is concerned with crossed products (skew products) by extended modular coactions associated with 1-cocycles having dense range. Such crossed products turn out to be factors. It is shown that the smooth flow of weights on such a crossed product is given by the skew-product action built from the original 1-cocycle.

We continue to use the notation from the preceding sections. For simplicity, we will denote by $\beta$ the coaction $\beta_{c}^{\phi}$ of $K$ on $N$ derived from a Borel 1-cocycle $c: \mathbb{R} \times X_{N} \rightarrow K$.

First we begin with a general lemma.

LEMMA 6.1. Let $\alpha$ be a coaction of $K$ on a von Neumann algebra $P$ acting on a Hilbert space $H$. If $D$ is a von Neumann subalgebra of $P^{\alpha}$, then the stabilization $\tilde{\alpha}$ satisfies $\left(B\left(L^{2}(K)\right) \otimes D\right)^{\tilde{\alpha}}=L^{\infty}(K) \otimes D$. 
Proof. Since $D$ is contained in $P^{\alpha}$, we have that, for any $T \in B\left(L^{2}(K)\right) \otimes D$, $\tilde{\alpha}(T)=\left(W_{K}\right)_{12} T_{23}\left(W_{K}\right)_{12}^{*}$. This shows that $\tilde{\alpha}$ restricts to $B\left(L^{2}(K)\right) \otimes D$. Moreover, this also implies that $T \in\left(B\left(L^{2}(K)\right) \otimes D\right)^{\tilde{\alpha}}$ if and only if $\left[T_{23},\left(W_{K}\right)_{12}\right]=0$, where $[a, b]:=a b-b a$. However, the latter condition is equivalent to $T$ belonging to $\left(L^{\infty}(K) \otimes \mathbb{C}\right)^{\prime}=L^{\infty}(K) \otimes B(H)$. Thus, we obtain the desired identity.

THEOREM 6.2. Suppose that the asymptotic range $r^{*}(c)$ of $c$ is equal to $K$. Then the relative commutant $\beta(N)^{\prime} \cap \hat{K}_{\beta} \ltimes N$ reduces to the scalar multiples of the identity. Namely, the coaction $\beta$ is strictly outer in the sense of [36].

Proof. Suppose that $T$ is an element of the relative commutant $\beta(N)^{\prime} \cap \hat{K}_{\beta} \ltimes N$. Since $N_{\phi}$ is included in $N^{\beta}$, it follows from the relative commutant theorem that we have

$T \in\left(\mathbb{C} \otimes N_{\phi}\right)^{\prime} \cap \hat{K}_{\beta} \ltimes N \subseteq B\left(L^{2}(K)\right) \otimes\left(N_{\phi}\right)^{\prime} \cap B\left(L^{2}(K)\right) \otimes N=B\left(L^{2}(K)\right) \otimes Z\left(N_{\phi}\right)$.

In the meantime, $T$ is in $\hat{K}_{\beta} \ltimes N=\left(B\left(L^{2}(K)\right) \otimes N\right)^{\tilde{\beta}}$. Hence, by Lemma 6.1, $T$ belongs to $L^{\infty}(K) \otimes Z\left(N_{\phi}\right)$. So $T$ can be regarded as a $\mathcal{U}\left(L^{\infty}(K)\right)$-valued Borel function on $X_{N}$, where $\mathcal{U}(P)$ in general stands for the unitary group of a von Neumann algebra $P$.

Next we consider the condition $\beta(u(s)) T \beta(u(s))^{*}=T$ for all $s \in \mathbb{R}$. Thus, $Q_{s}(1 \otimes u(s)) T\left(1 \otimes u(s)^{*}\right) Q_{s}^{*}=T$ for all $s \in \mathbb{R}$. This means that, for each $s \in \mathbb{R}$, we have

$$
\lambda_{K}(c(-s, x))^{*} T(-s x) \lambda_{K}(c(-s, x))=T(x) \quad\left(\text { for almost every } x \in X_{N}\right) .
$$

This can be written as an equation of functions on $K \times X_{N}$ as follows:

$$
T(c(-s, x) k,-s x)=T(k, x) \quad\left(\text { for almost every }(k, x) \in K \times X_{N}\right) .
$$

In order to understand this identity more transparently, we consider the ergodic measured groupoid $\mathcal{G}:=\mathbb{R} \times X_{N}$ and its skew product $\mathcal{G}_{c}$ by the 1-cocycle $c$ on $\mathcal{G}$. Recall that the groupoid structure on $\mathcal{G}_{c}:=K \times \mathcal{G}$ is defined as follows. The set $\mathcal{G}_{c}^{(2)}$ of multiplicative pairs consists of points of the form $(k,(s, t x)),\left(c(s, t x)^{-1} k,(t, x)\right)$, where $k \in K$ and $s, t \in \mathbb{R}$, $x \in X_{N}$. The product and the inverse are defined by

$$
\begin{gathered}
(k,(s, t x)),\left(c(s, t x)^{-1} k,(t, x)\right):=(k,(s+t, x)), \\
(k,(t, x))^{-1}:=\left(c(t, x)^{-1} k,(-t, t x)\right) .
\end{gathered}
$$

So the range map $r$ and the source map $s$ of $\mathcal{G}_{c}$ are respectively defined by $r(k,(t, x))=$ $(k, t x)$ and $s(k,(t, x))=\left(c(t, x)^{-1} k, x\right)$. Hence, the unit space of $\mathcal{G}_{c}$ is (identified with) $K \times X_{N}$. With this notation, (6.2) can be expressed as

$$
T(s(k,(t,-t x)))=T(r(k,(t,-t x))) .
$$

Since the cocycle $c$ satisfies $r^{*}(c)=K$, i.e. $c$ has dense range, the skew product $\mathcal{G}_{c}$ is ergodic (cf. [30, Corollaire II.3.5]). Therefore, $T$ must be constant.

In the rest of this section, assume that the asymptotic range $r^{*}(c)$ of $c$ is equal to $K$. Since the skew-product $K \times_{c} X_{N}$ is ergodic, it follows from [41, Theorem 3.1] that $K$ is necessarily amenable. Set $M:=\hat{K}_{\beta} \ltimes N$ and $\alpha:=\hat{\beta}$, the dual action of $\beta$ on $M$. So $\alpha$ is a minimal action of $K$ on $M$, due to Theorem 6.2. Namely, $\alpha$ is faithful and satisfies $\left(M^{\alpha}\right)^{\prime} \cap M=\mathbb{C}$. 
Our next aim is to completely describe the smooth flow of weights on $M$.

Let $\tilde{\phi}$ be the dual weight of $\phi$ on $M=\hat{K}_{\beta} \ltimes N$. Put $\tilde{M}:=\mathbb{R}_{\sigma} \tilde{\phi}^{\ltimes} M$. We denote by $\Theta_{\beta}$ the canonical extension of $\beta$ in the sense of [39, Definition 5.5]. So $\Theta_{\beta}$ is a coaction of $K$ on the crossed product $\tilde{N}:=\mathbb{R}_{\sigma^{\phi} \ltimes N} \cong B\left(L^{2}(\mathbb{R})\right) \otimes N_{\phi}$ (by the Takesaki duality). By Proposition 3.8 and [39, Theorem 4.1], the Radon-Nikodym derivative $(d \phi \circ \beta: d \phi)_{t}$ in the sense of [39] is equal to the identity. Hence, from [39, p. 542], the canonical extension $\Theta_{\beta}$ satisfies

$$
\begin{gathered}
\Theta_{\beta}\left(\sigma^{\phi}(a)\right)=\left(\operatorname{id}_{W^{*}(K)} \otimes \sigma^{\phi}\right)(\beta(a)) \quad(a \in N), \\
\Theta_{\beta}\left(\lambda_{\mathbb{R}}(s) \otimes 1\right)=1 \otimes \lambda_{\mathbb{R}}(s) \otimes 1 \quad(s \in \mathbb{R}),
\end{gathered}
$$

where, by abusing the notation, we let $\sigma^{\phi}$ denote the embedding of $N$ into $\tilde{N}$. For $a \in N_{\phi}$, we have $\sigma^{\phi}(a)=1 \otimes a$. We also have $\sigma^{\phi}(u(s))=f_{s} \otimes u(s)$ for any $s \in \mathbb{R}$. Here $f_{s}$ is the function on $\mathbb{R}$ given by $f_{s}(t):=e^{i s t}$. From these identities and Theorem 3.1, we obtain

$$
\begin{gathered}
\Theta_{\beta}(1 \otimes a)=1 \otimes 1 \otimes a \quad\left(a \in N_{\phi}\right), \\
\Theta_{\beta}\left(f_{s} \otimes u(s)\right)=\left(Q_{s}\right)_{13}\left(1 \otimes f_{s} \otimes u(s)\right) \quad(s \in \mathbb{R}) .
\end{gathered}
$$

In the meantime, thanks to [39, Proposition 5.7], $\widetilde{M}$ is isomorphic to $\hat{K}_{\Theta_{\beta}} \ltimes \tilde{N}$. Hence, from now on, we identify $\widetilde{M}$ with this crossed product. According to [39, Proposition 5.7] and (6.3)-(6.6), $\tilde{M}$ is generated by

$$
\underbrace{\left(\mathbb{C} \otimes \mathbb{C} \otimes N_{\phi}\right) \cup\left\{\left(Q_{s}\right)_{13}\left(1 \otimes f_{s} \otimes u(s)\right): s \in \mathbb{R}\right\} \cup\left(\mathbb{C} \otimes W^{*}(\mathbb{R}) \otimes \mathbb{C}\right)}_{\text {generate } \Theta_{\beta}(\tilde{N})}
$$

$$
\cup\left(L^{\infty}(K) \otimes \mathbb{C} \otimes \mathbb{C}\right)
$$

LEMMA 6.3. The fixed-point algebra $\tilde{N}^{\Theta}{ }^{\Theta}$ contains $W^{*}(\mathbb{R}) \otimes N_{\phi}$.

Proof. This follows from (6.4) and (6.5).

THEOREM 6.4. Under the situation considered above, we have the following.

(1) The center $Z(\tilde{M})$ of $\widetilde{M}$ is equal to $\Theta_{\beta}(\widetilde{N})^{\prime} \cap \widetilde{M}$, and $\widetilde{M}$ is generated by $\Theta_{\beta}(\tilde{N})$ and $Z(\tilde{M})$.

(2) The smooth flow of weights on $M$ consists of the flow space $X_{M}:=K \times X_{N}$ and the flow $F^{M}$ on $X_{M}$ given by $F_{t}^{M}(k, x):=(c(t, x) k, t x)$ for $t \in \mathbb{R}$ and $(k, x) \in K \times X_{N}$. The factor map from $X_{M}$ onto $X_{N}$ corresponding to the inclusion $Z(\tilde{N}) \subseteq Z(\tilde{M})$ is exactly the projection $(k, x) \in X_{M} \mapsto x \in X_{N}$.

(3) The Connes-Takesaki module $\bmod \left(\alpha_{k}\right)$ of $\alpha_{k}(k \in K)$ is given by

$$
\bmod \left(\alpha_{k}\right)(g, x)=\left(g k^{-1}, x\right) \quad\left(k \in K,(g, x) \in K \times X_{N}\right) .
$$

In particular, $\operatorname{Ker}(\bmod (\alpha))=\{e\}$.

Proof. (1) Take any element $T$ in the relative commutant $\Theta_{\beta}(\tilde{N})^{\prime} \cap \tilde{M}$. From the observation made above, we find

$$
\begin{aligned}
T & \in\left(\mathbb{C} \otimes W^{*}(\mathbb{R}) \otimes N_{\phi}\right)^{\prime} \cap B\left(L^{2}(K)\right) \otimes \tilde{N} \subseteq B\left(L^{2}(K)\right) \\
& \otimes\left\{\left(W^{*}(\mathbb{R}) \otimes N_{\phi}\right)^{\prime} \cap B\left(L^{2}(\mathbb{R})\right) \otimes N\right\} \\
& =B\left(L^{2}(K)\right) \otimes W^{*}(\mathbb{R}) \otimes\left(N_{\phi}\right)^{\prime} \cap N=B\left(L^{2}(K)\right) \otimes W^{*}(\mathbb{R}) \otimes Z\left(N_{\phi}\right) .
\end{aligned}
$$


From this, together with Lemmas 6.1 and 6.3 , it follows that $T$ belongs to $L^{\infty}(K) \otimes$ $W^{*}(\mathbb{R}) \otimes Z\left(N_{\phi}\right)$. This implies that $T$ commutes with every element of $L^{\infty}(K) \otimes \mathbb{C} \otimes \mathbb{C}$. Hence, $T$ is in $Z(\tilde{M})$. Thus, we have $Z(\tilde{M})=\Theta_{\beta}(\tilde{N})^{\prime} \cap \tilde{M}$. The assertion that $\widetilde{M}$ is generated by $\Theta_{\beta}(\tilde{N})$ and $Z(\widetilde{M})$ will be proven in part (2).

(2) For $T$ in part (1), we also have

$$
\left(Q_{s}\right)_{13}\left(1 \otimes f_{s} \otimes u(s)\right) T\left(1 \otimes f_{s}^{*} \otimes u(s)^{*}\right)\left(Q_{s}\right)_{13}^{*}=T
$$

for all $s \in \mathbb{R}$. By performing the Fourier transform on $\mathbb{R}$, we regard $T$ as a function in $L^{\infty}\left(K \times \mathbb{R} \times X_{N}\right)$. Then (6.7) is equivalent to the condition that, for every $s \in \mathbb{R}$,

$$
T(c(-s, x) k, u+s,-s x)=T(k, u, x) \quad\left(\text { for almost every }(k, u, x) \in K \times \mathbb{R} \times X_{N}\right) .
$$

On the measure space $K \times \mathbb{R} \times X_{N}$, define a measure-class-preserving flow $\left\{S_{t}\right\}$ by $S_{t}(k, u, x):=(c(-t, x) k, u+t,-t x)$. We denote by $\gamma: \mathbb{R} \rightarrow \operatorname{Aut}\left(L^{\infty}\left(K \times \mathbb{R} \times X_{N}\right)\right)$ the action of $\mathbb{R}$ induced by this flow $\left\{S_{t}\right\}$. By the result of the previous paragraph, we see that $Z(\tilde{M})$ is isomorphic to the fixed-point algebra $L^{\infty}\left(K \times \mathbb{R} \times X_{N}\right)^{\gamma}$. To realize the flow space of the smooth flow of weights on $M$, define a Borel surjective map $\psi: K \times \mathbb{R} \times X_{N} \rightarrow K \times X_{N}$ by

$$
\psi(k, u, x):=(c(u, x) k, u x) \quad\left((k, u, x) \in K \times \mathbb{R} \times X_{N}\right) .
$$

Note that 'the push-forward measure by $\psi$ ' is equivalent to the measure on $K \times X_{N}$ in question.

Claim. The map $\psi$ is an $\left\{S_{t}\right\}$-factor map (see [20, p. 11] for the term 'factor map'). In particular, $L^{\infty}\left(K \times X_{N}\right)$ is $*$-isomorphic to $L^{\infty}\left(K \times \mathbb{R} \times X_{N}\right)^{\gamma}$.

Proof of Claim. Let $f$ be a bounded Borel function on $K \times X_{N}$. Then

$$
\begin{aligned}
f \circ \psi(c(-s, x) k, u+s,-s x) & =f(c(u+s,-s x) c(-s, x) k,(u+s)(-s) x) \\
& =f(c(u, x) k, u x)=f \circ \psi(k, u, x) .
\end{aligned}
$$

This shows that $f \circ \psi$ is $\left\{S_{t}\right\}$-invariant.

Conversely, suppose that $F$ is a bounded Borel $\left\{S_{t}\right\}$-invariant function on $K \times \mathbb{R} \times X_{N}$. Set $\tilde{f}(k, x):=F(k, 0, x)$. Since $F$ is constant on the orbit $\left\{S_{t}(k, 0, x): t \in \mathbb{R}\right\}((k, x) \in$ $K \times X_{N}$ ), we easily find that $\tilde{f} \circ \psi=F$.

By the claim, $Z(\tilde{M})$ can be identified with $L^{\infty}\left(K \times X_{N}\right)$. The dual action $\left.\vartheta:=\widehat{(\sigma \tilde{\phi}}\right)$ of the dual weight $\tilde{\phi}$ on $M$ induces a flow $\left\{T_{t}\right\}$ on $K \times \mathbb{R} \times X_{N}$ given by $T_{t}(k, u, x)=$ $(k, u+t, x)$. Hence, the desired flow $\left\{F_{t}^{M}\right\}$ of weights on $M$ is given by $F_{t}^{M} \psi(k, u, x)=$ $\psi(k, u+t, x)$. Since

$$
\psi(k, u, x)=(c(u, x) k, u x), \quad \psi(k, u+t, x)=(c(t, u x) c(u, x) k, t(u x)),
$$

$\left\{F^{M}\right\}$ is actually given by $F_{t}^{M}(k, x)=(c(t, x) k, t x)$.

Now we show that $\widetilde{M}$ is generated by $\Theta_{\beta}(\tilde{N})$ and $Z(\tilde{M})$. For this, it is enough to prove that the von Neumann algebra generated by $\Theta_{\tilde{\alpha}}(\tilde{N})$ and $Z(\tilde{M})$ contains $L^{\infty}(K) \otimes$ $\mathbb{C} \otimes \mathbb{C}$. As we saw just before Lemma 6.3, $\Theta_{\beta}(\widetilde{N})$ contains $\mathbb{C} \otimes W^{*}(\mathbb{R}) \otimes Z\left(N_{\phi}\right)$. 
As in the previous paragraph, we assume that $\Theta_{\beta}(\tilde{N})$ contains $\mathbb{C} \otimes L^{\infty}(\mathbb{R}) \otimes Z\left(N_{\phi}\right)$ after performing the Fourier transform on $\mathbb{R}$. By the result of the preceding paragraph, we see that $Z(\tilde{M})$ and $\mathbb{C} \otimes L^{\infty}(\mathbb{R}) \otimes Z\left(N_{\phi}\right)$ together generate the von Neumann subalgebra $A$ of $L^{\infty}(K) \otimes L^{\infty}(\mathbb{R}) \otimes Z\left(N_{\phi}\right)$. Suppose that $\eta \in L^{1}\left(K \times \mathbb{R} \times X_{N}\right)$ satisfies

$$
\iiint \xi(k, s, x) \eta(k, s, x) d k d s d \mu(x)=0
$$

for any $\xi \in A$. In particular, we have

$$
\begin{aligned}
0 & =\iiint f(c(s, x) k) g(s) h(x) \eta(k, s, x) d k d s d \mu(x) \\
& =\iiint f(k) g(s) h(x) \eta\left(c(s, x)^{-1} k, s, x\right) d k d s d \mu(x)
\end{aligned}
$$

for any $f \in L^{\infty}(K), g \in L^{\infty}(\mathbb{R})$ and $h \in L^{\infty}\left(X_{N}\right)$. Hence, we obtain $\eta\left(c(s, x)^{-1} k, s, x\right)=0$ for almost every $(k, s, x) \in K \times \mathbb{R} \times X_{N}$. So

$$
0=\iiint\left|\eta\left(c(s, x)^{-1} k, s, x\right)\right| d k d s d \mu(x)=\iiint|\eta(k, s, x)| d k d s d \mu(x) .
$$

Thus, we conclude that $\eta=0$. It follows that $A$ coincides with $L^{\infty}\left(K \times \mathbb{R} \times X_{N}\right)$.

(3) Let $\tilde{\alpha}$ denote the canonical extension [19] of the action $\alpha$ to $\tilde{M}$. By [19], the ConnesTakesaki module $\bmod \left(\alpha_{k}\right)$ of $\alpha_{k}$ is just the restriction of $\tilde{\alpha}_{k}$ to $Z(\tilde{M})$.

By definition, $\tilde{\alpha}$ acts on $\tilde{M}$, regarded as being equal to $\mathbb{R}_{\sigma \tilde{\phi}} \ltimes M$ for the moment, as follows:

$$
\begin{gathered}
\tilde{\alpha}_{k}\left(\sigma^{\tilde{\phi}}(m)\right)=\sigma^{\tilde{\phi}}\left(\alpha_{k}(m)\right) \quad(m \in M), \\
\tilde{\alpha}_{k}\left(\lambda_{\mathbb{R}}(s) \otimes 1\right)=\delta_{K}(k)^{-i s} \sigma^{\tilde{\phi}}\left(\left(D \tilde{\phi} \circ \alpha_{k^{-1}}: D \tilde{\phi}\right)_{s}\right)\left(\lambda_{\mathbb{R}}(s) \otimes 1\right) \quad(s \in \mathbb{R}),
\end{gathered}
$$

where, as before, $\sigma^{\tilde{\phi}}$ stands for the embedding of $M$ into $\tilde{M}, \delta_{K}$ is the modular function of $K$, and $(D \omega: D v)_{t}$ in general indicates the Connes Radon-Nikodym cocycle for weights $\omega$ and $\nu$. Since $\tilde{\phi}$ is $\delta_{K}^{-1}$-invariant under the dual action $\tilde{\alpha}$ (see [35]), we have $\left(D \tilde{\phi} \circ \alpha_{k^{-1}}: D \tilde{\phi}\right)_{s}=\delta_{K}(k)^{i s}$. Thus,

$$
\tilde{\alpha}_{k}\left(\lambda_{\mathbb{R}}(s) \otimes 1\right)=\lambda_{\mathbb{R}}(s) \otimes 1 \quad(s \in \mathbb{R}) .
$$

Consequently, when $\tilde{M}$ is identified with $\hat{K}_{\Theta_{\beta} \ltimes} \widetilde{N}$, $\tilde{\alpha}$ fixes pointwise all elements in $\left(\mathbb{C} \otimes \mathbb{C} \otimes N_{\phi}\right) \cup\left\{\left(Q_{s}\right)_{13}\left(1 \otimes f_{s} \otimes u(s)\right): s \in \mathbb{R}\right\} \cup\left(\mathbb{C} \otimes W^{*}(\mathbb{R}) \otimes \mathbb{C}\right)$ and acts on $L^{\infty}(K) \otimes \mathbb{C} \otimes \mathbb{C}$ in the following manner: $\tilde{\alpha}_{k}(f \otimes 1 \otimes 1)=\rho_{k}(f) \otimes 1 \otimes 1$, where $\rho_{k}(f)(h):=f(h k)$. (In fact, $\tilde{\alpha}$ is just the dual action $\widehat{\Theta_{\beta}}$ of $\Theta_{\beta}$.) By the result of part (2), it is now easy to see that the Connes-Takesaki module $\bmod \left(\alpha_{k}\right)$ is given by the asserted equation.

COROLlARY 6.5. Keep the notation introduced so far. Then there exists a $*$-isomorphism $\Pi$ from $\tilde{M}$ onto $L^{\infty}(K) \otimes \tilde{N}$ such that:

(1) $\Pi \circ \tilde{\alpha}_{k}=\left(\operatorname{Ad} \rho_{K}(k) \otimes \mathrm{id}\right) \circ \Pi$;

(2) $\Pi(T)=1 \otimes T$ for all $T \in \tilde{N}$. 
Proof. By (the proof of) Theorem 6.4, we have a $K$-equivariant embedding $\pi$ of $L^{\infty}(K)$ into $Z(\tilde{M}) \subseteq \widetilde{M}$. From [28, Ch. II, §2], there exist a coaction $\delta_{0}$ of $K$ on $\widetilde{N}=\tilde{M}^{\tilde{\alpha}}$ and a $*$-isomorphism $\Pi$ from $\tilde{M}$ onto $\hat{K}_{\delta_{0}} \ltimes \tilde{N}$ such that: (i) $\Pi \circ \tilde{\alpha}_{k}=\left(\widehat{\delta_{0}}\right)_{k} \circ \Pi$ for all $k \in K$; (ii) $\Pi(T)=\delta_{0}(T)$ for all $T \in \widetilde{N}$; (iii) $\Pi(\pi(f))=f \otimes 1$ for all $f \in L^{\infty}(K)$. Since $L^{\infty}(K) \otimes \mathbb{C}=\Pi\left(\pi\left(L^{\infty}(K)\right)\right)$ is in the center of $\hat{K}_{\delta_{0}} \ltimes \widetilde{N}$, the coaction $\delta_{0}$ is trivial, i.e. $\delta_{0}(T)=1 \otimes T$ for any $T \in \widetilde{N}$. This means that $\hat{K}_{\delta_{0}} \ltimes \widetilde{N}=L^{\infty}(K) \otimes \widetilde{N}$. Thus, we obtain the assertion of this corollary.

7. Algebraic type of $\hat{K}_{\beta_{c}^{\phi}} \ltimes N$

This section is devoted to a study of the algebraic type of skew-product algebras obtained from extended modular coactions.

In this section, we assume from the beginning that the Borel 1-cocycle $c: \mathbb{R} \times X_{N} \rightarrow K$ has dense range. As we proved in $\S 6, \beta_{c}^{\phi}$ is strictly outer in this case. Hence, the crossed product $M:=\hat{K}_{\beta_{c}^{\phi}} \ltimes N$ is an infinite factor.

LEMMA 7.1. If $K$ is compact, then $M$ is factor of type III.

Proof. Since $K$ is compact, there exists a (unique) faithful normal conditional expectation

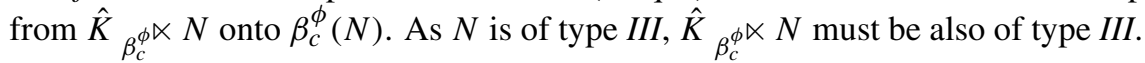

Suppose that $N$ is of type $I I I_{1}$. Since $Z\left(N_{\phi}\right)=\mathbb{C}$ in this case, $c$ is a Borel (hence, continuous) homomorphism from $\mathbb{R}$ into $K$ with $\overline{c(\mathbb{R})}=K$. So $K$ is so-called a solenoidal group. From [4, Propositions 5.15 and 5.16] or [26, pp. 87-89], it follows that $K$ is topologically isomorphic to $\mathbb{R}$ or a connected compact abelian group whose dual group is (isomorphic to) a subgroup of $\mathbb{R}$ with the discrete topology.

As we observed in Proposition 3.6, $\beta:=\beta_{c}^{\phi}$ is regarded as an action of the dual group $\hat{K}$ on $N$ by extended modular automorphisms. Since $N$ is of type $I I I_{1}$, the extended modular automorphisms are exactly the modular automorphisms. In this case, we have $\beta_{\gamma}=\sigma_{\hat{c}(\gamma)}^{\phi}$ for any $\gamma \in \hat{K}$, where $\hat{c}: \hat{K} \rightarrow \hat{\mathbb{R}}=\mathbb{R}$ is the dual map obtained from $c$.

Since $K$ is connected, $K$ cannot be discrete, unless $K$ is the trivial group, in which case $M=N$. Note that $K$ is trivial exactly when $\operatorname{Ker}(c)=\mathbb{R}$.

If $\operatorname{Ker}(c)$ is a proper closed subgroup of $\mathbb{R}$, then $\operatorname{Ker}(c)$ has the form $(-\log \lambda) \mathbf{Z}$ for a unique $\lambda$ with $0<\lambda<1$. In this case, since the quotient group $\mathbb{R} / \operatorname{Ker}(c)$ is compact, $c(\mathbb{R})$ must be compact as well. In particular, we have $c(\mathbb{R})=\overline{c(\mathbb{R})}=K$, and $K$ is thus topologically isomorphic to $\mathbb{T}$. We may assume in this case that $c$ is actually given by $c(t):=e^{2 \pi i t / \log \lambda}$. Then $\hat{c}(n)=2 \pi n / \log \lambda$, so we have $\beta_{n}=\left(\sigma_{T}^{\phi}\right)^{n}$, where $T=2 \pi / \log \lambda$. It follows from this that $M$ is of type $I I_{\lambda}$.

It remains to consider that case where $\operatorname{Ker}(c)=\{0\}$. As noted before, $K$ is either isomorphic to $\mathbb{R}$ or a connected compact abelian group whose dual group is (isomorphic to) a subgroup of $\mathbb{R}$ with the discrete topology. If $K$ is (isomorphic to) $\mathbb{R}$, then $\beta$ is nothing but the modular automorphism group $\sigma^{\phi}$. So $M$ is of type $I_{\infty}$. If $K$ is compact, then, by Lemma 7.1, $M$ is of type $I I I_{\lambda}$ for some $0 \leq \lambda<1$. From Theorem 6.4, the smooth flow $\left(F^{M}, X_{M}\right)$ of weights of $M$ is $\left(K,\left\{\xi_{t}\right\}\right)$, where $\xi_{t}(k):=k+c(t)$. If $M$ is of type $I I I_{\lambda}$ $(0<\lambda<1)$, then $\left(K,\left\{\xi_{t}\right\}\right)$ is conjugate to that $\left(\mathbb{T}, \zeta_{t}\right)$ given by $\zeta_{t}(z):=\exp (2 \pi i t / T) z$ $(z \in \mathbb{T})$, where $T=-\log \lambda$. Then, by Lemma B.1, $K$ and $\mathbb{T}$ are topologically isomorphic. 
It is clear in this case that $\operatorname{Ker}(c)$ is never trivial, which is a contradiction. So $M$ must be of type $I I I_{0}$, and $K$ is never isomorphic to $\mathbb{T}$.

To sum up, we have obtained the following.

Proposition 7.2. Suppose that $N$ is of type $I_{1} I_{1}$ (so that $c$ is a continuous homomorphism from $\mathbb{R}$ into $K$ ). Then $K$ must be either isomorphic to $\mathbb{R}$ or a connected compact abelian group whose dual group is (isomorphic to) a subgroup of $\mathbb{R}$ with the discrete topology. Moreover, only one of the following occurs.

(1) $K$ is the trivial group, and $M=N$.

(2) $\operatorname{Ker}(c)$ is a proper closed subgroup of $\mathbb{R}$ and $K$ is isomorphic to $\mathbb{T}$. In this case, $M$ is a factor of type $\operatorname{III}_{\lambda}(0<\lambda<1)$.

(3) The map c is a topological isomorphism, so that $K$ is isomorphic to $\mathbb{R}$. In this case, $M$ is of type $I_{\infty}$.

(4) $\operatorname{Ker}(c)$ is trivial, and $K$ is compact, but not isomorphic to $\mathbb{T}$. In this case, $M$ is of type $I I I_{0}$.

Proposition 7.3. Suppose that $M$ is of type $I_{\infty}$. Then $K$ is abelian and non-compact. Moreover:

(1) if $K$ is non-discrete as well, then $N$ is of type $I I I_{1}$;

(2) if $K$ is a discrete infinite group, then $N$ is of type $I_{\lambda}(0<\lambda<1)$.

Proof. By assumption, the smooth flow of weights on $M$ is the ergodic flow $\left(\mathbb{R},\left\{\eta_{t}\right\}\right)$, where $\eta_{t}(s):=s+t$. From Theorem 6.4 , we know that $\bmod (\alpha)$ is a faithful action of $K$ on $\mathbb{R}$ that commutes with $\left\{\eta_{t}\right\}$. Hence, there exists an injective continuous homomorphism $\chi$ from $K$ into $\mathbb{R}$ such that $\bmod \left(\alpha_{k}\right)=\eta_{\chi(k)}$ for any $k \in K$. By the injectivity of $\chi$, $K$ must be abelian. Moreover, since $\chi$ is a continuous homomorphism, $K$ must be noncompact. Note further that, since the smooth flow of weights on $M$ is transitive, the factor flow $\left(F^{N}, X_{N}\right)$ is also transitive. This means that $N$ is of type $I I I_{\lambda}(0<\lambda \leq 1)$.

If $K$ is further non-discrete, then $\overline{\chi(K)}=\mathbb{R}$. Since $L^{\infty}(\mathbb{R})^{\bmod (\alpha)}$ is isomorphic to $L^{\infty}\left(X_{N}\right)$ by Theorem 6.4, we have $L^{\infty}\left(X_{N}\right)=\mathbb{C}$. Therefore, $N$ is of type $I I I_{1}$.

Finally, if $K$ is a discrete infinite group, $N$ must be of type $I I I_{\lambda}(0<\lambda<1)$, because $N$ is never of type $I I I_{1}$ due to Proposition 7.2.

COROLlary 7.4. Suppose that $K$ is non-discrete and non-compact. Then the following are equivalent:

(1) $\quad \mathrm{N}$ is of type $\mathrm{III}_{1}$;

(2) $M$ is of type $I I_{\infty}$.

If one of the above conditions holds, then the cocycle $c$ is actually a topological group isomorphism from $\mathbb{R}$ onto $K$, and $M$ is (isomorphic to) the crossed product $\mathbb{R}_{\sigma \phi \ltimes N}$.

LEMMA 7.5. If $N$ is of type $I I I_{0}$, then so is $M$.

Proof. Since $\left(F^{N}, X_{N}\right)$ is a factor flow of $\left(F^{M}, X_{M}\right),\left(F^{M}, X_{M}\right)$ is properly ergodic if $\left(F^{N}, X_{N}\right)$ is. 
8. Minimal integrable actions on type III factors

In $\S 3$, we saw that each cocycle $c \in Z^{1}\left(F^{N}, K\right)$ determines, up to conjugacy, an action of $K$ on the skew-product algebra, i.e. the dual action of the extended modular coaction associated with $c$. The goal of this section is to give an abstract characterization of actions that arise in this way. This was done thoroughly by Izumi in [22] in the case of $K$ being compact. It turns out that the argument of Izumi can be largely extended to non-compact case under appropriate assumptions.

THEOREM 8.1. Let $\alpha$ be an integrable action of a locally compact group $K$ on a type III factor M. Suppose that the Connes-Takesaki module $\bmod (\alpha)$ of $\alpha$ is faithful and integrable. Then $\alpha$ is minimal, and the fixed-point algebra $M^{\alpha}$ is also a factor of type III. The smooth flow of weights on $M^{\alpha}$ is the factor flow of $F^{M}$ by $\bmod (\alpha)$. There exists a Borel 1 -cocycle $c$ in $Z^{1}\left(F^{M^{\alpha}}, K\right)$ having dense range in $K$, unique up to equivalence, such that $M$ is the skew product $L^{\infty}(K) \otimes_{c} M^{\alpha}$, and $\alpha$ is the dual action of the extended modular coaction associated with $c$. Moreover, $K$ is necessarily amenable.

Proof. Let $N:=M^{\alpha}$. Take a faithful normal semifinite weight $\phi$ on $N$ and put $\omega:=\phi \circ T_{\alpha}$. Set $\widetilde{M}:=\mathbb{R}_{\sigma \omega \ltimes} M \supseteq \widetilde{N}:=\mathbb{R}_{\sigma^{\phi} \ltimes} N$. Denote by $\theta$ the dual action of $\sigma^{\omega}$. As before, we write $\left(X_{M}, F^{M}\right)$ for the smooth flow of weights on $M$. We also denote by $\tilde{\alpha}$ the canonical extension of $\alpha$ to $\widetilde{M}$. Thus, we have

$$
\begin{aligned}
\tilde{\alpha}_{k}\left(\sigma^{\omega}(a)\right) & =\sigma^{\omega}\left(\alpha_{k}(a)\right) \quad(a \in M), \\
\tilde{\alpha}_{k}\left(\lambda_{\mathbb{R}}(s) \otimes 1\right) & =\delta_{K}(k)^{-i s} \sigma^{\omega}\left(\left(D \omega \circ \alpha_{k^{-1}}: D \omega\right)_{s}\right)\left(\lambda_{\mathbb{R}}(s) \otimes 1\right) \quad(s \in \mathbb{R}),
\end{aligned}
$$

where, as before, $\sigma^{\omega}$ again stands for the embedding of $M$ into $\tilde{M}$. We know [19] that $\bmod (\alpha)$ is just the restriction of $\tilde{\alpha}$ to $Z(\tilde{M})$. We also have $\widetilde{N}=\tilde{M}^{\tilde{\alpha}}$.

Let us choose a standard Borel probability measure space $(Y, v)$ and an ergodic flow $\left\{F_{t}\right\}_{t \in \mathbb{R}}$ such that $Z(\tilde{M})^{\bmod (\alpha)}=L^{\infty}(Y, v)$ and $\theta_{t}(f)=f \circ F_{-t}$ for $f \in L^{\infty}(Y, v)$ and $t \in \mathbb{R}$. Thus, we have a canonical $\mathbb{R}$-equivariant Borel map from $X_{M}$ onto $Y$ such that $p_{*}(\mu) \sim v$, that is, $p: X_{M} \rightarrow Y$ is an ergodic extension of $Y$ to $X_{M}$. From [11, Proposition 1], it follows that there exist a standard Borel space $S$, a probability measure $m$ on $S$, a Borel isomorphism $\Phi: S \times Y \rightarrow X_{M}$ and a Borel cocycle $a: \mathbb{R} \times Y \rightarrow$ $\operatorname{Aut}\left(L^{\infty}(S, m)\right)$, where $\operatorname{Aut}\left(L^{\infty}(S, m)\right)$ is equipped with the usual (Polish group) topology when $L^{\infty}(S, m)$ is viewed as a von Neumann algebra, such that:

(1) $\Phi_{*}(m \times v) \sim \mu$;

(2) $p \circ \Phi(s, y)=y$ for almost every $\in y \in Y$;

(3) for each $t \in \mathbb{R}$, one has $\Phi\left(a(t, y)(s), F_{t}(y)\right)=F_{t}^{M}(\Phi(s, y))$ for almost every $(s, y) \in S \times Y$.

Hence, we may assume from the outset that $\left(X_{M}, \mu\right)$ is $(S \times Y, m \times v)$ and $F^{M}$ is given by $F_{t}^{M}(s, y)=\left(a(t, y)(s), F_{t}(y)\right)$. By construction, the module action $\bmod (\alpha)$ induces an ergodic action $\left\{T_{k}\right\}_{k \in K}$ of $K$ on $S$ satisfying $T_{k} \circ a(t, y)=a(t, y) \circ T_{k}$. (More precisely, $T_{k}$ is determined by the identity: $\Phi_{*} \circ \bmod \left(\alpha_{k}\right) \circ\left(\Phi_{*}\right)^{-1}(f \otimes 1)=$ $f \circ T_{k^{-1}} \otimes 1$ for $f \in L^{\infty}(S, m)$, where $\Phi_{*}$ is a $*$-isomorphism from $L^{\infty}\left(X_{M}\right)$ onto $L^{\infty}(S \times Y)$ induced by $\Phi$.) The integrability of $\bmod (\alpha)$ implies that of $\left\{T_{k}\right\}$. From this and [33, Ch. X, Lemma 4.13], we may and do assume that there is a compact subgroup $H$ of $K$ such that $S=H \backslash K$ and $T_{k}$ is the right translation action of $K$ on $S=H \backslash K$. 
Since $\operatorname{Ker}(\bmod (\alpha))=\{e\}$, we find that $H$ must be trivial. So $S=K$ and $T_{k}$ is the right translation action of $K$ on $K$. Since $a(t, y)$ commutes with the right translations $\left\{T_{k}\right\}$, it follows from [34, Theorem 1] that there is a Borel 1-cocycle $c: \mathbb{R} \times Y \rightarrow K$ such that $a(t, y)(k)=c(t, y) k$ for $k \in K$. Therefore, we conclude that $X_{M}$ is the skew product $K \times Y$ of $Y$ by the 1-cocycle $c$, and that $F^{M}$ is nothing but the skew-product action. Note that $c$ has dense range, because $F^{M}$ is ergodic.

By the previous paragraph, there is an embedding $\pi$ of $L^{\infty}(K)$ into $Z(\tilde{M})$ such that $\pi \circ \operatorname{Ad} \rho_{K}(k)=\tilde{\alpha}_{k} \circ \pi$ for all $k \in K$. From [28, Ch. II, §2], we find that there are a coaction $\gamma$ of $K$ on $\widetilde{N}$ and a $*$-isomorphism $\Pi_{1}$ from $\widetilde{M}$ onto $\hat{K} \gamma \ltimes \widetilde{N}$ such that: (i) $\Pi_{1} \circ \tilde{\alpha}=\hat{\gamma} \circ \Pi_{1}$; (ii) $\Pi_{1}(T)=\gamma(T)$ for all $T \in \tilde{N}$; (iii) $\Pi_{1}(\pi(f))=f \otimes 1$ for any $f \in L^{\infty}(K)$. Since $L^{\infty}(K) \otimes \mathbb{C}=\Pi_{1}\left(\pi\left(L^{\infty}(K)\right)\right)$ is contained the center of $\hat{K} \gamma \ltimes \tilde{N}$, $\gamma$ must be trivial, i.e. $\gamma(T)=1 \otimes T$ for any $T \in \widetilde{N}$. Thus, we get $\Pi_{1}(\tilde{M})=L^{\infty}(K) \otimes \widetilde{N}$. From this, we see that $\widetilde{N}^{\prime} \cap \tilde{M}=Z(\tilde{M})$ and $\widetilde{M}=\left(\tilde{M} \cap \tilde{N}^{\prime}\right) \vee \widetilde{N}$. By the first identity, we get $Z(\tilde{N})=\left(\tilde{N}^{\prime} \cap \tilde{M}\right)^{\tilde{\alpha}}=Z(\tilde{M})^{\bmod (\alpha)}=L^{\infty}(Y, v)$. Since $\theta$ acts on $Z(\tilde{M})^{\bmod (\alpha)}$ ergodically, $N$ must be a factor. In fact, we can do more. By the results obtained above, we have

$$
\begin{aligned}
\Pi_{1}\left(\tilde{M} \cap \sigma^{\phi}(N)^{\prime}\right) & =\left(L^{\infty}(K) \otimes \tilde{N}\right) \cap\left(\mathbb{C} \otimes \sigma^{\phi}(N)\right)^{\prime}=L^{\infty}(K) \otimes\left(\tilde{N} \cap \sigma^{\phi}(N)^{\prime}\right) \\
& =L^{\infty}(K) \otimes Z(\tilde{N})=\Pi_{1}(Z(\tilde{M})) .
\end{aligned}
$$

Hence, $\tilde{M} \cap \sigma^{\phi}(N)^{\prime}=Z(\tilde{M})$. So, if $a \in M \cap N^{\prime}$, then $\sigma^{\phi}(a) \in Z(\tilde{M})$. Since $\theta$ acts on $Z(\tilde{M})^{\bmod (\alpha)}$ ergodically, $a$ must be a scalar. Therefore, we obtain $M \cap N^{\prime}=\mathbb{C}$. If $N$ were semifinite, then we may assume that $\phi$ is a trace, in which case $Z(\tilde{N})$ is equal to $W^{*}(\mathbb{R}) \otimes \mathbb{C}$. Thus, $\left(Y, \mathbb{R}, F_{t}\right)$ is conjugate to the translation of $\mathbb{R}$ on $\mathbb{R}$. This would entail that $c$ is cohomologous to the trivial cocycle. Since $\left(X_{M}, F^{M}\right)$ is ergodic, it would then follow that $K$ is trivial, which implies that $M=N$, a contradiction. Hence, $N$ is a type III factor. We now see that the smooth flow $\left(X_{N}, F^{N}\right)$ of weights on $N$ is $(Y, F)$ defined above.

We now assume that the weight $\phi$ which we started with is a dominant weight on $N$.

Since $c$ is a Borel 1-cocycle on the smooth flow of weights $\left(X_{N}, F^{N}\right)$, we may consider the extended modular coaction $\beta:=\beta_{c}^{\phi}$ of $K$ on $N$ associated with $c$. Let $L:=\hat{K}_{\beta} \ltimes N=L^{\infty}(K) \otimes_{c} N$ and $\eta$ the dual weight of $\phi$. The crossed product $\widetilde{L}:=\mathbb{R}_{\sigma \eta \ltimes L}$ is, as before, identified with $\hat{K}_{\Theta_{\beta}} \ltimes \tilde{N}$. Denote by $\vartheta^{L}$ the dual action of $\sigma^{\eta}$. We write $T_{1}$ for the operator-valued weight associated with the dual action $\widehat{\left(\Theta_{\beta}\right)}$ : $T_{1}(a)=\int_{K}{\widehat{(\Theta \beta})_{k}}_{(a)} d k$. Note that the restriction $E_{1}$ of $T_{1}$ to $\tilde{M} \cap \Theta_{\beta}(\tilde{N})^{\prime}=Z(\tilde{M})$ is still semifinite.

In the meantime, we have found that $\alpha$ is an integrable, minimal action with type III fixed-point algebra $N$. From [35, Proposition 6.4], it follows that $\alpha$ is dual. Hence, there exists a strictly outer coaction $\tau$ of $K$ on $N$ such that $(M, \alpha)$ is conjugate to $\left(\hat{K}_{\tau} \ltimes N, \hat{\tau}\right)$. In the following, we identify $(M, \alpha)$ with $\left(\hat{K}_{\tau} \ltimes N, \hat{\tau}\right)$. So the weight $\omega$ on $M$ introduced before is the dual weight of $\phi$. The inclusion $(\widetilde{M} \supseteq \widetilde{N})$ should

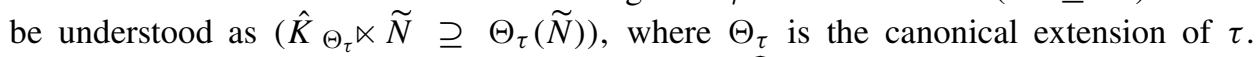
The canonical extension $\tilde{\alpha}$ of $\alpha$ is the dual action $\widehat{\left(\Theta_{\tau}\right)}$. Denote by $\vartheta^{M}$ the dual action of $\sigma^{\omega}$. We write $T_{2}$ for the operator-valued weight associated with the dual action $\widehat{\left(\Theta_{\tau}\right)}$ : 
$T_{2}(a)=\int_{K}{\widehat{\left(\Theta_{\tau}\right)_{k}}}_{k}(a) d k$. Note that the restriction $E_{2}$ of $T_{2}$ to $\tilde{M} \cap \Theta_{\tau}(\tilde{N})^{\prime}=Z(\tilde{M})$ is semifinite, since $\bmod (\alpha)=\left.\widetilde{\left(\Theta_{\tau}\right)_{k}}\right|_{Z(\widetilde{M})}$ is integrable by assumption.

By Theorem 6.4 and the results obtained above, there exists a $*$-isomorphism $\Phi_{0}$ from $Z(\tilde{M})$ onto $Z(\tilde{L})$ such that $\Phi_{0}(a)=a$ for any $a \in Z(\tilde{N})$ (more precisely, $\Phi_{0}\left(\Theta_{\tau}(a)\right)=\Theta_{\beta}(a)$ for all $\left.a \in Z(\tilde{N})\right),\left.\Phi_{0} \circ \vartheta_{t}^{M}\right|_{Z(\tilde{M})}=\left.\vartheta_{t}^{L}\right|_{Z(\widetilde{L})} \circ \Phi_{0}$ for all $t \in \mathbb{R}$ and $\Phi_{0} \circ \bmod \left(\alpha_{k}\right)={\widehat{\left(\Theta_{\beta}\right)}}_{k} \circ \Phi_{0}$ for any $k \in K$. From the last identity, we have $E_{1}\left(\Phi_{0}(a)\right)=\Phi_{0}\left(E_{2}(a)\right)\left(=E_{2}(a)\right)$ for $a \in Z(\tilde{M})_{+}$.

Take a faithful normal state $\chi$ on $\tilde{N}$ and put $\chi_{1}:=\chi \circ T_{1}, \chi_{2}:=\chi \circ T_{2}$. Let $\mathfrak{n}_{1}$ be the linear span of the elements of the form $a b$, where $a \in \widetilde{N}$ and $b \in \mathfrak{n}_{E_{1}}$. Set $\mathfrak{m}_{1}:=\mathfrak{n}_{1}^{*} \mathfrak{n}_{1}$. Then $\mathfrak{m}_{1}$ is a $*$-subalgebra of $\mathfrak{n}_{\chi_{1}} \cap \mathfrak{n}_{\chi_{1}}^{*}$ which is $\sigma$-strongly* dense in $\widetilde{L}$ and globally invariant under the modular automorphism group $\sigma^{\chi_{1}}$. By [23, Lemma 2.1], $\Lambda_{\chi_{1}}\left(\mathfrak{m}_{1}\right)$ is dense in $H_{\chi_{1}}$.

As in the previous paragraph, let $\mathfrak{n}_{2}$ be the linear span of elements $a b\left(a \in \widetilde{N}, b \in \mathfrak{n}_{E_{2}}\right)$ and $\mathfrak{m}_{2}$ be $\mathfrak{n}_{2}^{*} \mathfrak{n}_{2}$. Then $\Lambda_{\chi_{2}}\left(\mathfrak{m}_{2}\right)$ is dense in $H_{\chi_{2}}$.

Now define a linear operator $V_{0}$ from $\Lambda_{\chi_{2}}\left(\mathfrak{n}_{2}\right)$ into $\Lambda_{\chi_{1}}\left(\mathfrak{n}_{1}\right)$ by

$$
V_{0} \Lambda_{\chi_{2}}\left(\sum_{i=1}^{n} a_{i} b_{i}\right):=\Lambda_{\chi_{1}}\left(\sum_{i=1}^{n} a_{i} \Phi_{0}\left(b_{i}\right)\right) \quad\left(a_{i} \in \tilde{N}, b_{i} \in \mathfrak{n}_{E_{2}}\right) .
$$

Since $\Phi_{0}\left(\mathfrak{n}_{E_{2}}\right)=\mathfrak{n}_{E_{1}}$, the left-hand side of the equality above makes sense. We have

$$
\begin{aligned}
\left\|\Lambda_{\chi_{1}}\left(\sum_{i=1}^{n} a_{i} \Phi_{0}\left(b_{i}\right)\right)\right\|^{2} & =\sum_{i, j=1}^{n} \chi \circ T_{1}\left(a_{i}^{*} \Phi_{0}\left(b_{i}^{*} b_{j}\right) a_{j}\right)=\sum_{i, j=1}^{n} \chi\left(a_{i}^{*} E_{1}\left(\Phi_{0}\left(b_{i}^{*} b_{j}\right)\right) a_{j}\right) \\
& =\sum_{i, j=1}^{n} \chi\left(a_{i}^{*} E_{2}\left(b_{i}^{*} b_{j}\right) a_{j}\right)=\sum_{i, j=1}^{n} \chi \circ T_{2}\left(a_{i}^{*} b_{i}^{*} b_{j} a_{j}\right) \\
& =\left\|\Lambda_{\chi 2}\left(\sum_{i=1}^{n} a_{i} b_{i}\right)\right\|^{2} .
\end{aligned}
$$

This shows that $V_{0}$ is an isometry. It is clearly surjective. Hence, it can be extended to a unitary $V$ from $H_{\chi_{2}}$ onto $H_{\chi_{1}}$. It is easy to check that one has $V a b V^{*}=a \Phi_{0}(b)$ for any $a \in \widetilde{N}$ and $b \in Z(\widetilde{M})$. From this and the fact that $\widetilde{M}=\widetilde{N} \vee Z(\widetilde{M}), \widetilde{L}=\widetilde{N} \vee Z(\widetilde{L})$, it follows that the equation

$$
\Phi(a):=\operatorname{VaV}^{*} \quad(a \in \tilde{M})
$$

defines a $*$-isomorphism from $\tilde{M}$ onto $\widetilde{L}$ satisfying $\Phi(a)=a$ for all $a \in \widetilde{N}$ and $\Phi(b)=\Phi_{0}(b)$ for all $b \in Z(\tilde{M})$. It is now obvious that we have $\Phi \circ \vartheta_{t}^{M}=\vartheta_{t}^{L} \circ \Phi$ for any $t \in \mathbb{R}$. As $M=\widetilde{M}^{\vartheta^{M}}$ and $L=\widetilde{L}^{\vartheta^{L}}, M$ is $*$-isomorphic to $L$ through $\Phi$. So we identify $M$ with $L$.

We now have two integrable, minimal actions $\alpha$ and $\hat{\beta}$ of $K$ on $M$, both of which have the same fixed-point algebra $N$. From [38, Theorem 3.6], we find that $\operatorname{Aut}\left(M / M^{\hat{\beta}}\right)$, the group of automorphisms of $M$ leaving $M^{\alpha}$ pointwise invariant, is exactly $\left\{\hat{\beta}_{k}: k \in K\right\}=$ $\left\{\alpha_{k}: k \in K\right\}$. Hence, there exists a (topological) group automorphism $\ell$ of $K$ such that 


$$
\begin{aligned}
& \hat{\beta}_{k}=\alpha_{\ell(k)} \text { for any } k \in K \text {. If } k \in K, \text { then } \\
& \begin{aligned}
\bmod \left(\alpha_{k^{-1} \ell(k)}\right) \\
\quad=\bmod \left(\alpha_{k^{-1}} \circ \alpha_{\ell(k)}\right)=\bmod \left(\alpha_{k^{-1}}\right) \circ \bmod \left(\hat{\beta}_{k}\right) \\
=(\text { the right multiplication on } K \text { by } k) \circ\left(\text { the right multiplication on } K \text { by } k^{-1}\right) \\
=\text { id. }
\end{aligned}
\end{aligned}
$$

Since $\bmod (\alpha)$ is faithful, we obtain $k^{-1} \ell(k)=e$, i.e. $\ell(k)=k$. Therefore, $\alpha=\hat{\beta}$.

Finally, since the skew product $X_{M}=K \times_{c} X_{N}$ is ergodic, we find from [41, Theorem 3.1] that $K$ is amenable.

COROLLARY 8.2. Let $M$ be an AFD factor of type III. For every (necessarily amenable) locally compact subgroup $K$ of $\operatorname{Aut}\left(F^{M}\right)$ which acts integrably, there exists an integrable, minimal action $\alpha$ of $K$ on $M$ such that $\bmod \left(\alpha_{k}\right)=k$ for any $k \in K$. Such an $\alpha$ is unique up to conjugacy in the sense that, if $\alpha^{1}$ is another integrable, minimal action of $K$ on $M$ with $\bmod \left(\alpha_{k}^{1}\right)=k$ for any $k \in K$, then there exists $a *$-automorphism $v$ of $M$ such that $\alpha_{k}^{1}=v \circ \alpha_{k} \circ v^{-1}$ for all $k \in K$.

Proof. Let $K$ be a non-trivial locally compact group as above. Choose a standard Borel probability space $(Y, \mu)$ such that $L^{\infty}\left(X_{M}, \mu_{M}\right)^{K}=L^{\infty}(Y, \mu)$. Thus, we have an ergodic extension $X_{M} \rightarrow Y$ of $\mathbb{R}$-spaces. Then, as in the second paragraph of the proof of Theorem 8.1, there exists a Borel 1-cocycle $c: \mathbb{R} \times Y \rightarrow K$ with dense range such that $X_{M}=K \times{ }_{c} Y$ and

$$
F_{t}^{M}(k, y)=(c(t, y) k, t y), \quad \bmod \left(\alpha_{l}\right)(k, y)=\left(k l^{-1}, y\right) .
$$

Note that the $\mathbb{R}$-space $Y$ is never conjugate to the translation of $\mathbb{R}$ on $\mathbb{R}$. Take the AFD factor $N$ of type $I I I$ whose smooth flow of weights is the $\mathbb{R}$-space $Y$. Hence, $c$ belongs to $Z^{1}\left(F^{N}, K\right)$.

Fix a dominant weight $\phi$ on $N$ and consider the extended modular coaction $\beta:=\beta_{c}^{\phi}$ associated with $c$. Set $P:=L^{\infty}(K) \otimes_{c} N$ and $\alpha:=\hat{\beta}$. Denote by $\omega$ the dual weight of $\phi$. Since $N$ is AFD, $\widetilde{N}=\mathbb{R}_{\sigma^{\phi} \ltimes N}$ is also AFD. By Corollary 6.5, $\widetilde{P}:=\mathbb{R}_{\sigma^{\omega} \ltimes P}$ is again AFD. In particular, $P$ is AFD. In the meantime, by Theorem 6.4 , both $M$ and $P$ have the same smooth flow of weights. Therefore, they are isomorphic $[\mathbf{6 - 8 , 1 8 , 2 4 ]}$. So we may assume that $M=L^{\infty}(K) \otimes_{c} N$. Now, by Theorem 6.4, we may take $\alpha$ for the desired action.

Let $\alpha^{1}$ be another integrable, minimal action of $K$ on $M$ with $\bmod \left(\alpha_{k}^{1}\right)=k$ for any $k \in K$. From Theorem 8.1, it follows that there exists a cocycle $c_{1} \in Z^{1}\left(F^{N}, K\right)$ such that $M=L^{\infty}(K) \otimes_{c_{1}} N$ and $\alpha^{1}=\widehat{\left(\beta_{c_{1}}^{\phi}\right)}$. Then $K \times_{c} X_{N}$ and $K \times_{c_{1}} X_{N}$ are isomorphic $\mathbb{R}$-spaces over $X_{N}$. From this, it follows that $c$ is cohomologous to $c_{1}$. By Proposition 3.3, $\beta$ is cocycle conjugate to $\beta_{c_{1}}^{\phi}$. Hence, the dual actions $\alpha$ and $\alpha^{1}$ are conjugate.

\section{Remark on Galois correspondence}

This section is concerned with Galois correspondence for such actions as treated in the previous section. Namely, for such an action $\alpha$ of a locally compact group $K$ on a type $I I I$ 
factor $M$, we consider the problem of deciding which intermediate subfactor $L$ of the inclusion $M^{\alpha} \subseteq M$ has the form $L=M^{\alpha_{H}}$ for a closed subgroup $H$ of $K$. It is shown with some conditions that $L$ is of the form $L=M^{\alpha_{H}}$ exactly when there exist faithful normal semifinite operator-valued weights from $M$ to $L$ and from $L$ to $M^{\alpha}$.

Throughout this section, we fix an integrable, minimal action $\alpha$ of an amenable locally compact group $K$ on a factor $M$ of type III satisfying a condition that the module $\bmod (\alpha)$ of $\alpha$ is faithful and integrable. Set $N:=M^{\alpha}$. Thanks to Theorem $8.1, N$ is a factor of type $I I I$ and we may assume that there exists a Borel 1-cocycle $c \in Z^{1}\left(F^{N}, K\right)$ such that $M=L^{\infty}(K) \otimes_{c} N$ and $\alpha$ is the dual action $\hat{\beta}$ of the extended modular coaction $\beta:=\beta_{c}^{\phi}$ associated with $c$ and a dominant weight $\phi$ on $N$. Remark that, due to (the proof of) [35, Proposition 6.4], $\alpha$ is a dominant action, i.e. $\alpha$ is conjugate to the stabilized action $\operatorname{Ad} \rho_{K}(\cdot) \otimes \alpha$ on $B\left(L^{2}(K)\right) \otimes M$.

As in $\S 3$, let $\{u(s)\}$ be a one- parameter unitary group of $N$ satisfying $\sigma_{t}^{\phi}(u(s))=$ $e^{-i s t} u(s)$ for all $s, t \in \mathbb{R}$, and put $\theta_{s}:=\left.\operatorname{Ad} u(s)\right|_{N_{\phi}}$. Denote by $\tau$ the faithful normal semifinite trace on $N_{\phi}$ with $\tau \circ \theta_{s}=e^{-s} \tau$ for any $s \in \mathbb{R}$.

LEMMA 9.1. Let $L$ be an intermediate subfactor of $N \subseteq M$. Then there exists a closed subgroup $H$ of $K$ such that $L \cap\left(L^{\infty}(K) \otimes N_{\phi}\right)=L^{\infty}(K / H) \otimes N_{\phi}$.

Proof. Thanks to Proposition C.3, we know that the covariant system

$$
\left(L^{\infty}(K) \otimes N_{\phi}, \mathbb{R},\left.\operatorname{Ad} \beta_{c}^{\phi}(u(s))\right|_{L^{\infty}(K) \otimes N_{\phi}}, \tau_{K} \otimes \tau\right)
$$

gives a continuous decomposition of $M$. For each $t \in \mathbb{T}$, set $\kappa_{t}:=\left.\operatorname{Ad} \beta_{c}^{\phi}(u(s))\right|_{L^{\infty}(K) \otimes N_{\phi}}$. Note (see Proposition C.3) that the restriction of $\kappa$ to $L^{\infty}(K) \otimes Z\left(N_{\phi}\right)=L^{\infty}\left(K \times X_{N}\right)$ is the skew-product action induced from the cocycle $c$.

Since $\beta_{c}^{\phi}(u(t))$ belongs to $L$ for all $t \in \mathbb{R}, Q:=L \cap\left(L^{\infty}(K) \otimes N_{\phi}\right)$ is left globally invariant under the action $\kappa$. Put $A:=L \cap\left(L^{\infty}(K) \otimes Z\left(N_{\phi}\right)\right)$. Take a standard Borel probability space $(Y, v)$ such that $A=L^{\infty}(Y, v)$. By considering $\left.\kappa_{t}\right|_{A}$ for any $t \in \mathbb{R},(Y, v)$ can be regarded as an intermediate ergodic $\mathbb{R}$-space of the (normal) ergodic extension $K \times{ }_{c} X_{N} \rightarrow X_{N}$. From [12, Theorem 1.4], it follows that there exists a closed subgroup $H$ of $K$ such that $Y$ is isomorphic to $K / H \times_{c} X_{N}$ over $X_{N}$. Hence, we assume from the outset that $Y$ is $K / H \times{ }_{c} X_{N}$. Thus, $A=L^{\infty}(K / H) \otimes Z\left(N_{\phi}\right)$. In particular, $Q$ contains $L^{\infty}(K / H) \otimes N_{\phi}$. From this, it is easy to see that the restriction of the trace $\tau_{0} \otimes \tau$ to $Q$ is still semifinite, where $\tau_{0}$ is a normal state on $L^{\infty}(K)$ induced from a Borel probability measure on $K$. Hence, there exists a unique faithful normal conditional expectation $E_{Q}$ from $L^{\infty}(K) \otimes N_{\phi}$ onto $Q$ such that $\left(\tau_{0} \otimes \tau\right) \circ E_{Q}=\tau_{0} \otimes \tau$. Let $f \in L^{\infty}(K)$ and $a \in N_{\phi}$. Since $\mathbb{C} \otimes N_{\phi}=\beta_{c}^{\phi}\left(N_{\phi}\right)$ is contained in $L$, we have that $1 \otimes a \in Q$. So

$$
(1 \otimes a) E_{Q}(f \otimes 1)=E_{Q}(f \otimes a)=E_{Q}(f \otimes 1)(1 \otimes a) .
$$

From this, we get

$$
E_{Q}(f \otimes 1) \in Q \cap\left(\mathbb{C} \otimes N_{\phi}\right)^{\prime}=L \cap\left(L^{\infty}(K) \otimes Z\left(N_{\phi}\right)\right)=L^{\infty}(K / H) \otimes Z\left(N_{\phi}\right) .
$$

Thanks to this, we see that $Q=E_{Q}\left(L^{\infty}(K) \otimes N_{\phi}\right)$ is included in $L^{\infty}(K / H) \otimes N_{\phi}$. Thus, we are done. 
Definition 9.2. Let $\beta$ be a coaction of a locally compact group $G$ on a von Neumann algebra $P$. According to [27], the equation

$$
\beta^{d}(X):=\operatorname{Ad}\left(W_{K}\right)_{12}(1 \otimes X) \quad\left(X \in \widehat{G}_{\beta} \ltimes P\right)
$$

defines a coaction $\beta^{d}$ of $G$ on $\widehat{G}{ }_{\beta} \ltimes P$.

THEOREM 9.3. For an intermediate subfactor $L$ of $M^{\alpha} \subseteq M$, the following are equivalent.

(1) $L$ is globally invariant under the coaction $\beta^{d}$, i.e. $\beta^{d}(L) \subseteq W^{*}(K) \otimes L$.

(2) $L$ is generated by $L^{\infty}(K / H) \otimes \mathbb{C}$ and $\beta\left(M^{\alpha}\right)$ for some closed subgroup $H$ of $K$.

(3) $L=M^{\alpha_{H}}$ for some closed subgroup $H$ of $K$.

(4) L is globally invariant under the modular automorphism group of the dual weight of $\phi$.

If one (hence, all) of the above conditions holds, then the closed subgroup $H$ is determined uniquely by (2) or (3) or by Lemma 9.1.

Moreover, if the homogeneous space $K / H$ admits a $K$-invariant Borel measure for the subgroup $H$ of $K$ which $L$ determines by Lemma 9.1, then the above conditions (1)-(4) are also equivalent to:

(5) there exist faithful normal operator-valued weights $E: M \rightarrow L$ and $F: L \rightarrow M^{\alpha}$.

In this case, L is of type III and the smooth flow of weights on $L$ is given by the skewproduct action on $\mathrm{K} / \mathrm{H} \times_{c} X_{N}$.

Proof. The equivalence of (1) and (2) is due to [28, Ch. VII, Theorem 2.2]. The equivalence of (2) and (3) is due to [28, Ch. VII, Theorem 1.2].

(3) $\Rightarrow$ (4) Let $\omega$ be the dual weight of $\phi$. By Proposition 3.8 and [39, Lemma 2.1], we have $\sigma_{t}^{\omega}(f \otimes 1)=f \otimes 1$ for all $f \in L^{\infty}(K)$ and all $t \in \mathbb{R}$. Moreover, by [35, Proposition 3.7], we have $\sigma_{t}^{\omega}\left(\beta\left(M^{\alpha}\right)\right)=\beta\left(M^{\alpha}\right)$ for any $t \in \mathbb{R}$. Meanwhile, by (2), $L$ is generated by $L^{\infty}(K / H) \otimes \mathbb{C}$ and $\beta\left(M^{\alpha}\right)$. It is now clear that $L$ is globally invariant under $\sigma^{\omega}$.

(4) $\Rightarrow$ (3) Consider the closed subgroup $H$ of $K$ obtained by Lemma 9.1. For any $t \in \mathbb{R}$, put $\gamma_{t}:=\left.\sigma_{t}^{\omega}\right|_{L}$, where $\omega$ is the dual weight of $\phi$. Since $M_{\omega}=L^{\infty}(K) \otimes N_{\phi}$ by Corollary C.4, it follows that $L^{\gamma}=M_{\omega} \cap L=L^{\infty}(K / H) \otimes N_{\phi}$. Moreover, by [35, Proposition 3.7], we have

$$
\gamma_{s}(\beta(u(t)))=\sigma_{s}^{\omega}(\beta(u(t)))=\beta\left(\sigma_{s}^{\phi}(u(t))\right)=e^{-i s t} \beta(u(t))
$$

for any $s, t \in \mathbb{R}$. From [28, Ch. II, §2], $\gamma$ is a dual action. In particular, we get

$$
\begin{aligned}
L & =L^{\gamma} \vee\{\beta(u(t)): t \in \mathbb{R}\}^{\prime \prime}=L^{\infty}(K / H) \otimes N_{\phi} \vee\{\beta(u(t)): t \in \mathbb{R}\}^{\prime \prime} \\
& =L^{\infty}(K / H) \otimes \mathbb{C} \vee \beta(N) .
\end{aligned}
$$

By [28, Ch. VII, Theorem 1.2], the last term in the above identities is equal to $M^{\alpha_{H}}$.

Therefore, the conditions (1)-(4) are all equivalent.

Let $H$ be the subgroup of $K$ determined by $L$ through Lemma 9.1, and suppose from now on that the homogeneous space $K / H$ admits a $K$-invariant Borel measure $\mu$. 
(3) $\Rightarrow$ (5): Suppose that $L=M^{\alpha_{H}}$. Since $\alpha$ is dominant, the equation

$$
E(a):=\int_{H} \alpha_{k}(a) d k \quad\left(a \in M_{+}\right)
$$

defines a faithful normal semifinite operator-valued weight $E$ from $M$ to $L$.

Define a unitary operator $S$ on $L^{2}(K) \otimes L^{2}(K)$ by

$$
\{S \xi\}(k, l):=\delta(k)^{1 / 2} \xi(k, l k) \quad\left(\xi \in L^{2}(K \times K)\right) .
$$

Note that, for any $f \in L^{\infty}(K), S(1 \otimes f) S^{*}$ is a function in $L^{\infty}(K) \otimes L^{\infty}(K)$ given by $(k, l) \in K \times K \mapsto f(l k)$. So, if $f \in L^{\infty}(K / H)$, then $S(1 \otimes f) S^{*}$ belongs to $L^{\infty}(K / H) \otimes L^{\infty}(K)$. Meanwhile, because $S$ belongs to $L^{\infty}(K) \otimes W^{*}(K)^{\prime}$, we have $S_{12}(1 \otimes \beta(a)) S_{12}^{*}=1 \otimes \beta(a)$ for all $a \in N$. Since $L=L^{\infty}(K / H) \otimes \mathbb{C} \vee \beta(N)$ by the implication $(3) \Rightarrow(2)$, it follows from the above observation that the equation

$$
\eta(X):=(S \otimes 1)(1 \otimes X)\left(S^{*} \otimes 1\right) \quad(X \in L)
$$

defines an injective normal unital $*$-homomorphism from $L$ into $L^{\infty}(K / H) \otimes M$. Take any $X \in L_{+}$and put $F(X):=\left(\mu \otimes \operatorname{id}_{M}\right)(\eta(X))$. We claim that $F(X)$ belongs to the extended positive part of $M^{\alpha}=\beta(N)$. For this, it suffices to show that $F(X)$ is fixed by the dual action $\alpha$. First we observe that $S$ satisfies $\left(\lambda_{K}(k) \otimes \rho_{K}(k)\right) S=S\left(\lambda_{K}(k) \otimes 1\right)$ for any $k \in K$. Since $\mu \circ \operatorname{Ad} \lambda_{K}(k)=\mu$ by the K-invariance of $\mu$, we have

$$
\begin{aligned}
\alpha_{k}(F(X)) & =\left(\mu \otimes \operatorname{id}_{M}\right)\left(\left(\operatorname{Ad} \lambda_{K}(k) \otimes \alpha_{k}\right)(\eta(X))\right) \\
& =\left(\mu \otimes \operatorname{id}_{M}\right)\left(\operatorname{Ad}\left(\left(\lambda_{K}(k) \otimes \rho_{K}(k)\right) S \otimes 1\right)(1 \otimes X)\right) \\
& =\left(\mu \otimes \operatorname{id}_{M}\right)\left(\operatorname{Ad}\left(S\left(\lambda_{K}(k) \otimes 1\right) \otimes 1\right)(1 \otimes X)\right) \\
& =\left(\mu \otimes \operatorname{id}_{M}\right)\left(\operatorname{Ad} S_{12}(1 \otimes X)\right) \\
& =\left(\mu \otimes \operatorname{id}_{M}\right)(\eta(X))=F(X) .
\end{aligned}
$$

Thus our claim has been proven. Because $\eta(\beta(a))=1 \otimes \beta(a)$ for all $a \in N$, we have

$$
F\left(\beta(a)^{*} X \beta(a)\right)=\beta(a)^{*} F(X) \beta(a) .
$$

Hence we find that $F$ is a faithful normal operator-valued weight from $L$ onto $M^{\alpha}$. If $f$ is a positive function in $L^{\infty}(K / H) \cap L^{1}(K / H)$, then, by the $K$ - invariance of $\mu$, we have

$$
F(f \otimes 1)=\left(\int_{K / H} f d \mu\right) \cdot 1 .
$$

This shows that $F$ is semifinite.

(5) $\Rightarrow(3)$ : Put $N:=M^{\alpha}$. By assumption, we have two faithful normal semifinite operator-valued weights $T_{\alpha}$ and $F \circ E$ from $M$ onto $N$. Note that the Radon-Nikodym cocycle $\left[D T_{\alpha}: D(F \circ E)\right]_{t}$ belongs to $N^{\prime} \cap M=\mathbb{C}$. So $T_{\alpha}$ is a positive scalar multiple of $F \circ E$. Hence, we may assume that $T_{\alpha}=F \circ E$.

Set $\omega:=\phi \circ T_{\alpha}$ and $\psi:=\phi \circ F$. 
Let

$$
\tilde{M}:=\mathbb{R}_{\sigma^{\omega} \ltimes M}, \quad \widetilde{L}:=\mathbb{R}_{\sigma^{\psi} \ltimes L}, \quad \tilde{N}:=\mathbb{R}_{\sigma^{\phi} \ltimes N} .
$$

Then both $\widetilde{L}$ and $\widetilde{N}$ are regarded as von Neumann subalgebras of $\widetilde{M}$ satisfying $\widetilde{N} \subseteq$ $\widetilde{L} \subseteq \widetilde{M}$. As we saw in the proof of Theorem 6.4 , we have $\widetilde{M}=\widetilde{N} \vee Z(\widetilde{M})$ and $\widetilde{N}^{\prime} \cap \tilde{M}=Z(\widetilde{M})$. Moreover, we know that the smooth flow $\left(X_{M}, F^{M}\right)$ of weights on $M$ is the $\mathbb{R}$-ergodic extension $\left(K \times_{c} X_{N}, m_{K} \times \mu_{N}\right)$, the skew product by $c$, over the smooth flow $\left(X_{N}, F^{N}\right)$ of weights on $N$. Since $Z(\tilde{M}) \supseteq Z(\widetilde{L}) \supseteq Z(\tilde{N})$, the smooth flow $\left(X_{L}, F^{L}\right)$ of weights on $L$ is an intermediate factor space of the extension $K \times_{c} X_{N} \rightarrow X_{N}$. From [12, Theorem 1.4], there exists a closed subgroup $H$ of $K$ such that $X_{L}$ is (isomorphic to) the (so-called isometric) extension $K / H \times_{c} X_{N}$ over $X_{N}$ : $F_{t}^{L}(q(k), x)=\left(q(c(t, x) k), F_{t}^{N} x\right)$ for $(q(k), x) \in K / H \times X_{N}$, where $q: K \rightarrow K / H$ is the quotient map. This particularly means that $Z(\widetilde{L})$ is exactly the fixed-point algebra $Z(\tilde{M})^{\bmod \left(\alpha_{H}\right)}=L^{\infty}(K / H) \otimes L^{\infty}\left(X_{N}\right)$, and that the smooth flow of weights on $L$ is given by the skew-product action on $K / H \times_{c} X_{N}$. Note that the skew-product action on $K / H \times{ }_{c} X_{N}$ is never conjugate to ( $\mathbb{R}$, Translation), since $M$ is of type III. In particular, $L$ must be of type III.

Claim. $\widetilde{L}$ is generated by $\widetilde{N}$ and $Z(\widetilde{L})$. Moreover, we have $\widetilde{L}=\widetilde{M}^{\tilde{\alpha}_{H}}$, where $\tilde{\alpha}$ is the canonical extension of $\alpha$ to $\tilde{M}$. (As noted before, $\tilde{\alpha}$ can be identified with the dual action $\widehat{\Theta_{\beta}}$ of the canonical extension $\Theta_{\beta}$ of the coaction $\beta$ to $\widetilde{N}$.)

Proof of Claim. Let $\vartheta^{M}$ be the dual action $\widehat{\left.\sigma^{\omega}\right)}$, and put $\vartheta^{L}:=\left.\vartheta^{M}\right|_{\tilde{L}}, \vartheta^{N}:=\left.\vartheta^{M}\right|_{\tilde{N}}$. Denote by $\tau_{\omega}$ the faithful normal semifinite trace on $\widetilde{M}$ satisfying $\tau_{\omega} \circ \vartheta_{t}^{M}=e^{-t} \tau_{\omega}$ for any $t \in \mathbb{R}$. Define $\tau_{\psi}$ and $\tau_{\phi}$ similarly. Take a unique faithful normal semifinite operator-valued weight $\widetilde{E}$ from $\widetilde{M}$ to $\widetilde{L}$ such that $\tau_{\omega}=\tau_{\psi} \circ \widetilde{E}$. Choose also a unique operator-valued weight $\widetilde{F}$ from $\widetilde{L}$ to $\widetilde{N}$ with $\tau_{\psi}=\tau_{\phi} \circ \widetilde{F}$. So $\widetilde{F} \circ \widetilde{E}$ is a faithful normal semifinite operator-valued weight from $\widetilde{M}$ to $\widetilde{N}$. Meanwhile, we have an operator-valued weight $T_{\tilde{\alpha}}$ from $\widetilde{M}$ to $\widetilde{N}$ associated with the cannonical extension $\tilde{\alpha}$, which is semifinite due to the integrability of $\alpha$. Moreover, the restriction of $T_{\tilde{\alpha}}$ to $\tilde{M} \cap \widetilde{N}^{\prime}=Z(\tilde{M})$ is still semifinite, because of the integrability of the module action $\bmod (\alpha)$. By [17], the restriction of $\widetilde{F} \circ \widetilde{E}$ to $Z(\widetilde{M})$ is semifinite. In particular, $S:=\left.\widetilde{\widetilde{E}}\right|_{Z(\widetilde{M})_{+}}$is a faithful normal semifinite operator-valued weight from $Z(\tilde{M})$ to $Z(\tilde{L})$. By [17], the $\sigma$-weak closure of $S\left(\mathfrak{m}_{S}\right)$ coincides with $Z(\widetilde{L})$. Let $\mathfrak{n}_{0}$ be the linear span of elements of the form $a b$, where $a \in \widetilde{N}$ and $b \in \mathfrak{n}_{S}$. Since $\widetilde{M}=\widetilde{N} \vee Z(\widetilde{M})$, it follows that $\mathfrak{n}_{0}$ is a $\sigma$-strongly* dense left ideal of $\tilde{M}$. Put $\mathfrak{m}_{0}=\mathfrak{n}_{0}^{*} \mathfrak{n}_{0}$. Then $\mathfrak{m}_{0}$ is also $\sigma$-weakly dense in $\widetilde{M}$. From this, it is easily checked that the $\sigma$-weak closure of $\widetilde{E}\left(\mathfrak{m}_{0}\right)$ coincides with $\widetilde{L}$. Note that, by definition, $\widetilde{E}\left(\mathfrak{m}_{0}\right)$ is the subspace generated by $\widetilde{N} S\left(\mathfrak{m}_{S}\right)$. Therefore, $\widetilde{L}$ is generated by $\widetilde{N}$ and $Z(\widetilde{L})$.

Let $\Pi$ be the $*$-isomorphism from $\tilde{M}$ onto $L^{\infty}(K) \otimes \widetilde{N}$. By the result of the previous paragraph, we see that $\Pi(\widetilde{L})$ is generated by $\Pi(\widetilde{N})=\mathbb{C} \otimes \widetilde{N}$ and $\Pi(Z(\widetilde{L}))$. Since $Z(\widetilde{L})=$ $Z(\tilde{M})^{\bmod \left(\alpha_{H}\right)}=Z(\tilde{M})^{\tilde{\alpha}_{H}}$, it follows that

$$
\begin{aligned}
\Pi(Z(\tilde{L})) & =\Pi(Z(\tilde{M}))^{\left\{\operatorname{Ad}\left(\rho_{K}(k) \otimes 1\right): k \in H\right\}}=\left(L^{\infty}(K) \otimes Z(\tilde{N})\right)^{\left\{\operatorname{Ad}\left(\rho_{K}(k) \otimes 1\right): k \in H\right\}} \\
& =L^{\infty}(K / H) \otimes Z(\tilde{N}) .
\end{aligned}
$$


Hence,

$$
\begin{aligned}
\Pi(\tilde{L}) & =L^{\infty}(K / H) \otimes \tilde{N}=\left(L^{\infty}(K) \otimes \tilde{N}\right)^{\left\{\operatorname{Ad}\left(\rho_{K}(k) \otimes 1\right): k \in H\right\}} \\
& =\Pi(\tilde{M})^{\left\{\operatorname{Ad}\left(\rho_{K}(k) \otimes 1\right): k \in H\right\}}=\Pi\left(\tilde{M}^{\tilde{\alpha}_{H}}\right) .
\end{aligned}
$$

Therefore, we obtain $\widetilde{L}=\tilde{M}^{\tilde{\alpha}_{H}}$. Thus, the claim has been proven.

By the claim, we have

$$
L=\widetilde{L}^{\vartheta^{L}}=\left(\tilde{M}^{\tilde{\alpha}_{H}}\right)^{\vartheta^{M}}=\left(\widetilde{M}^{\vartheta^{M}}\right)^{\tilde{\alpha}_{H}}=M^{\alpha_{H}} .
$$

Thus we are done.

Remark. We conclude this section with a remark on Theorem 9.3. If $K$ is compact, then, by [23, Theorem 3.15], there always exists a (unique) faithful normal conditional expectation from $M$ onto an arbitrary intermediate subfactor containing $N:=M^{\alpha}$. So one gets a complete Galois correspondence in this case. Hence, there might always be a faithful normal semifinite operator-valued weight from $M$ to any intermediate subfactor, even if $K$ is no longer compact. However, we do not know whether this is the case or not. It would be very interesting to give a complete answer to this problem. If we set about finding a solution to this problem, we see that it suffices to examine the case in which both $M$ and $M^{\alpha}$ are of type $I I I_{0}$. Indeed, since $K$ may be assumed to be non-trivial, i.e. not equal to $\{e\}$, it follows from Theorem 6.4 that it is enough to treat the case where $M$ is of type $I I I_{\lambda}$ $(\lambda \neq 1)$. In that case, due to Proposition $7.2, N$ being of type $I I_{1}$ entails that $K$ must be compact. Hence, [23] takes care of the complete Galois correspondence when $N$ is a type $I I I_{1}$ subfactor. So we may suppose that $N$ is also of type $I I I_{\lambda^{\prime}}\left(0 \leq \lambda^{\prime}<1\right)$.

Assume for the moment that $M$ is a type $I I I_{\lambda}(0<\lambda<1)$ factor. Then, by [9, Ch. IV, Proposition 1.3], Aut $\left(F^{M}\right)$ is a compact (abelian) group, isomorphic to $\mathbb{T}$. So the closure $\overline{\bmod \left(\alpha_{K}\right)}$ of $\bmod \left(\alpha_{K}\right)$ in $\operatorname{Aut}\left(F^{M}\right)$ is either a finite group or $\operatorname{Aut}\left(F^{M}\right)$ itself. In the former case, $K$ is a finite group, as $\bmod (\alpha)$ is faithful. Hence, [23] takes care of the Galois correspondence in this case again. In the latter case, we have

$$
P_{N}=P_{M}^{\bmod \left(\alpha_{K}\right)}=P_{M}^{\operatorname{Aut}\left(F^{M}\right)}=\mathbb{C} .
$$

This means that $N$ is of type $I I I_{1}$, a contradiction.

It thus remains to investigate the case where $M$ is of type $I I I_{0}$. Assume for a while that $N$ is of type $I I I_{\lambda^{\prime}}\left(0<\lambda^{\prime}<1\right)$. Then $X_{N}=\left[0,-\log \lambda^{\prime}\right)$ and $F_{t}^{N} s=s+t \bmod \left(-\log \lambda^{\prime}\right)$. Note that, since $P_{N} \subseteq P_{M}$ and $F_{t}^{N}=\left.F_{t}^{M}\right|_{P_{N}}$ for any $t \in \mathbb{R}$, the $T$-set $T(M)$ (see [5]) contains the $T$-set

$$
T(N)=\frac{2 \pi}{\log \lambda^{\prime}} \mathbf{Z} .
$$

Let $\xi_{0}(x):=\exp \left(-2 \pi i x / \log \lambda^{\prime}\right)\left(0 \leq x<-\log \lambda^{\prime}\right)$, which is an eigenfunction for the flow $F^{N}$ corresponding to

$$
-\frac{2 \pi}{\log \lambda^{\prime}} \in T(N)
$$

Let $\pi: X_{M}=K \times X_{N} \rightarrow X_{N}$ be the projection and set $\eta_{0}:=\xi_{0} \circ \pi$. It is clear that $\eta_{0}$ is, in turn, an eigenfunction for the flow $F^{M}$. Put

$$
\Omega:=\left\{(k, x) \in X_{M}: \eta_{0}(k, x)=1\right\} .
$$


It follows at once that $\Omega=K \times\{0\}$, so we may identify $\Omega$ with $K$. We have

$$
F_{-\log \lambda^{\prime}}^{M}(k, 0)=\left(c\left(-\log \lambda^{\prime}, 0\right) k, F_{-\log \lambda^{\prime}}^{N} 0\right)=\left(c\left(-\log \lambda^{\prime}, 0\right) k, 0\right) .
$$

So we define a transformation $T$ on $\Omega=K$ by $T k:=c\left(-\log \lambda^{\prime}, 0\right) k$ for any $k \in K$. Then the flow $\left(X_{M}, F^{M}\right)$ is realized as the flow built under the constant function $-\log \lambda^{\prime}$ with the base transformation $T$ on $\Omega=K$. Since $\Omega=K$ must be non-atomic and $T$ is ergodic, we find that $K$ is non-discrete, and that the closure of $\left\{c\left(-\log \lambda^{\prime}, 0\right)^{n}: n \in \mathbf{Z}\right\}$ is dense in $K$. Hence, $K$ is a so-called monothetic group. By [29, Theorem 2.3.2] (or [4, Theorem 19, p. 11, 25]), $K$ is a compact abelian group. So, once again, [23] takes care of the complete Galois correspondence.

Therefore, it suffices to examine the case in which both $M$ and $M^{\alpha}$ are of type $I I I_{0}$, as claimed.

\section{A. Appendix. Borel unitary cocycles}

The unitary 1-cocycles for group actions on von Neumann algebras are assumed to be $\sigma$-strongly* continuous with respect to the group parameters. Here we show that the $\sigma$-strong* continuity requirement can be replaced, without modifying the notion, by the measurability condition as follows. Our proof relies upon the argument in [5].

LEMMA A.1. Let $\alpha$ be an action of a locally compact group $G$ on a von Neumann algebra $M$. Suppose that $u: G \rightarrow \mathcal{U}(M)$ is a Borel map satisfying $u_{g h}=u_{g} \alpha_{g}\left(u_{h}\right)$ for any $g, h \in$ $G$, where $\mathcal{U}(M)$ is the unitary group of $M$ equipped with the strong operator topology. Then there is a unitary $U$ in $M \otimes L^{\infty}(G)$ such that $u_{g} \otimes 1=U\left(\alpha_{g} \otimes \operatorname{Ad} \lambda_{G}(g)\right)\left(U^{*}\right)$ for all $g \in G$.

Proof. Take a Hilbert space $H$ for which $\{M, H\}$ is a standard representation. Define a unitary $U$ on $H \otimes L^{2}(G)=L^{2}(G, H)$ by $\{U \xi\}(g):=u_{g} \xi(g)$. Since $U$ can be regarded as an $M$-valued bounded Borel function on $G$ given by $g \in G \mapsto u_{g} \in M, U$ belongs to $M \otimes L^{\infty}(G) \subseteq M \otimes B\left(L^{2}(G)\right)$. Let $v(g)$ be the canonical implementation of $\alpha_{g}$ on $H$. Then, for any $\xi \in L^{2}(G, H)$, we have

$$
\begin{aligned}
\left\{\left(\alpha_{g} \otimes \operatorname{Ad} \lambda_{G}(g)\right)(U) \xi\right\}(h) & =\left\{\left(v_{g} \otimes \lambda_{G}(g)\right) U\left(v(g)^{*} \otimes \lambda_{G}(g)^{*}\right) \xi\right\}(h) \\
& =v(g)\left\{U\left(v(g)^{*} \otimes \lambda_{G}(g)^{*}\right) \xi\right\}\left(g^{-1} h\right) \\
& =v(g) u_{g^{-1} h}\left\{\left(v(g)^{*} \otimes \lambda_{G}(g)^{*}\right) \xi\right\}\left(g^{-1} h\right) \\
& =v(g) u_{g^{-1} h} v(g)^{*} \xi(h)=\alpha_{g}\left(u_{g^{-1} h}\right) \xi(h) \\
& =\alpha_{g}\left(u_{g^{-1}} \alpha_{g^{-1}}\left(u_{h}\right)\right) \xi(h) \\
& =\alpha_{g}\left(u_{g^{-1}}\right) u_{h} \xi(h)=u_{g}^{*} u_{h} \xi(h) \\
& =\left\{\left(u_{g}^{*} \otimes 1\right) U \xi\right\}(h) .
\end{aligned}
$$

Thus, we get $\left(\alpha_{g} \otimes \operatorname{Ad} \lambda_{G}(g)\right)(U)=\left(u_{g}^{*} \otimes 1\right) U$. This completes the proof.

Thanks to Lemma A.1, we immediately obtain the announced assertion below.

COROLlARY A.2. Let $\alpha$ be an action of a locally compact group $G$ on a von Neumann algebra $M$. The set of all unitary $\alpha-1$-cocycles coincides with that of all Borel maps $u: G \rightarrow \mathcal{U}(M)$ satisfying $u_{g h}=u_{g} \alpha_{g}\left(u_{h}\right)$ for any $g, h \in G$. 
Let $M$ be an infinite factor. We denote by $\left(P_{M}, \mathbb{R}, \mathcal{F}^{M}\right)$ be the smooth flow of weights on $M$. Then there exist a standard Borel probability space $\left(X_{M}, \mu_{M}\right)$ and a flow $\left\{F_{t}^{M}\right\}_{t \in \mathbb{R}}$ on $X_{M}$ such that $P_{M}=L^{\infty}\left(X_{M}, \mu_{M}\right)$ and $\mathcal{F}_{t}^{M}(h)=h \circ F_{-t}^{M}$ for any $h \in L^{\infty}\left(X_{M}, \mu_{M}\right)$ and any $t \in \mathbb{R}$. Following convention, let $Z_{\mathcal{F}^{M}}^{1}\left(\mathbb{R}, \mathcal{U}\left(P_{M}\right)\right)$ stand for the set of all unitary $\mathcal{F}^{M}$-1-cocycles on $P_{M}$.

Let $c \in Z^{1}\left(F^{M}, \mathbb{T}\right)$. For any $t \in \mathbb{R}$, define a bounded Borel function $u_{t}^{c}$ on $X_{M}$ by $u_{t}^{c}(x):=c(-t, x)$. Then we have

$$
\begin{aligned}
u_{s+t}^{c}(x) & =c(-s-t, x)=c\left(-t, F_{-s}^{M} x\right) c(-s, x) \\
& =u_{s}^{c}(x) u_{t}^{c}\left(F_{-s}^{M} x\right)=u_{s}^{c}(x) \mathcal{F}_{s}^{M}\left(u_{t}^{c}\right)(x) .
\end{aligned}
$$

Hence, $u_{s+t}^{c}=u_{s}^{c} \mathcal{F}_{s}^{M}\left(u_{t}^{c}\right)$.

Lemma A.3. The map $t \in \mathbb{R} \mapsto u_{t}^{c} \in \mathcal{U}\left(P_{M}\right)$ is $\sigma$-strongly* continuous. Therefore, $u^{c}$ is a unitary $\mathcal{F}^{M}$-1-cocycle on $P_{M}=L^{\infty}\left(X_{M}\right)$.

Proof. The idea of our proof is more or less the same as that of Lemma A.1. Define a unitary $U$ on the Hilbert space $L^{2}\left(\mathbb{R} \times X_{M}\right)$ by $\{U \xi\}(s, x):=c(-s, x) \xi(s, x)$. It is easy to see that $U$ belongs to $L^{\infty}(\mathbb{R}) \otimes L^{\infty}\left(X_{M}\right)$. Let $t \in \mathbb{R}$. Then we have

$$
\begin{aligned}
\left\{U\left(\operatorname{Ad} \lambda_{\mathbb{R}}(t) \otimes \mathcal{F}_{t}^{M}\right)\left(U^{*}\right) \xi\right\}(s, x) & =c(-s, x) \overline{c\left(-s+t, F_{-t}^{M} x\right)} \xi(s, x) \\
& =c(-s, x) \overline{c(-s, x) c\left(t, F_{-t}^{M} x\right)} \xi(s, x) \\
& =c(-t, x) \xi(s, x)=\left\{\left(1 \otimes u_{t}^{c}\right) \xi\right\}(s, x) .
\end{aligned}
$$

Thus, we obtain $U\left(\operatorname{Ad} \lambda_{\mathbb{R}}(t) \otimes \mathcal{F}_{t}^{M}\right)\left(U^{*}\right)=1 \otimes u_{t}^{c}$. From this, the $\sigma$-strong* continuity of $u^{c}$ follows.

Proposition A.4. The map

$$
c \in Z^{1}\left(F^{M}, \mathbb{T}\right) \longmapsto u^{c} \in Z_{\mathcal{F}^{M}}^{1}\left(\mathbb{R}, \mathcal{U}\left(P_{M}\right)\right)
$$

introduced above establishes a bijective correspondence between $Z^{1}\left(F^{M}, \mathbb{T}\right)$ and $Z_{\mathcal{F}^{M}}^{1}\left(\mathbb{R}, \mathcal{U}\left(P_{M}\right)\right)$.

Proof. It is clear that the map $c \mapsto u^{c}$ is injective.

Let $u \in Z_{\mathcal{F} M}^{1}\left(\mathbb{R}, \mathcal{U}\left(P_{M}\right)\right)$. Define a unitary $U$ on the Hilbert space $L^{2}\left(\mathbb{R} \times X_{M}\right)$ by $\{U \xi\}(s, x):=u_{s}(x) \xi(s, x)$. By the proof of Lemma A.1, we have $1 \otimes u_{t}=$ $U\left(\operatorname{Ad} \lambda_{\mathbb{R}}(t) \otimes \mathcal{F}_{t}^{M}\right)\left(U^{*}\right)$ for all $t \in \mathbb{R}$. Since $U$ belongs to $L^{\infty}(\mathbb{R}) \otimes L^{\infty}\left(X_{M}\right)$, it follows that there exists a bounded Borel function $f$ on $\mathbb{R} \times X_{M}$ with $|f|=1$ such that $U$ is the multiplication operator induced by $f$. Define a function $c^{\prime}: \mathbb{R} \times X_{M} \rightarrow \mathbb{T}$ by $c^{\prime}(s, x):=f(-s, x)$. By the definition of $U$, we find that, for each $t \in \mathbb{R}$, $u_{t}(x)=c^{\prime}(-t, x)$ for almost every $x \in X_{M}$. Meanwhile, by the cocycle property of $u$, we have $u_{s+t}=u_{s} \cdot\left(u_{t} \circ F_{-s}^{M}\right)$ for any $s, t \in \mathbb{R}$. From this, it follows that, for each $s, t \in \mathbb{R}$, one has $c^{\prime}(-s-t, x)=c^{\prime}(-s, x) c^{\prime}\left(-t, F_{-s}^{M} x\right)$ for almost every $x \in X_{M}$. Hence, from [42, Theorem B.9], there exists a cocycle $c \in Z^{1}\left(F^{M}, \mathbb{T}\right)$ such that $c=c^{\prime}$ almost everywhere. Now it is easy to check that $u^{c}=u$. 
B. Appendix. Isomorphism

LEMMA B.1. Let $H, K$ be locally compact abelian groups with left Haar measures $m_{H}$ and $m_{K}$, respectively. Suppose that there are continuous homomorphisms $p: \mathbb{R} \rightarrow H$ and $q: \mathbb{R} \rightarrow K$ with dense ranges such that the ergodic flows $\xi_{t}(g):=g+p(t)$ and $\eta_{t}(k):=k+q(t)$ are conjugate. Then there is a topological group isomorphism $\pi: H \rightarrow K$ such that $\pi \circ p=q$.

Proof. Let $\Phi$ be a Borel isomorphism from $H$ onto $K$ such that $m_{K} \circ \Phi \sim m_{H}$ and, for each $t \in \mathbb{R}, \Phi \circ \xi_{t}(h)=\eta_{t} \circ \Phi(h)$ for almost every $h \in H$, and $\Phi^{-1} \circ \eta_{t}(k)=\xi_{t} \circ \Phi^{-1}(k)$ for almost every $k \in K$.

Define a unitary $W: L^{2}(H) \rightarrow L^{2}(K)$ by

$$
\{W \zeta\}(k):=\sqrt{\frac{d m_{H} \circ \Phi^{-1}}{d m_{K}}(k) \zeta\left(\Phi^{-1}(k)\right) \quad\left(\zeta \in L^{2}(H)\right) .}
$$

Then we have $W \lambda_{H}(p(t)) W^{*}=\lambda_{K}(q(t))$ for all $t \in \mathbb{R}$. From this and the fact that $\overline{p(\mathbb{R})}=H$ and $\overline{q(\mathbb{R})}=K$, it follows that $W \lambda_{H}(H) W^{*}=\lambda_{K}(K)$. Hence, the equation

$$
W \lambda_{H}(h) W^{*}=\lambda_{K}(\pi(h)) \quad(h \in H)
$$

defines a topological group isomorphism $\pi$ from $H$ onto $K$. It is clear that we have $\pi(p(t))=q(t)$ for all $t \in \mathbb{R}$.

C. Appendix. Another construction of $L^{\infty}(K) \otimes_{c} N$

Let $N$ be a factor of type $I I I$ and $\phi$ a dominant weight on $N$. Also, let $\{u(s)\}_{s \in \mathbb{R}} \subseteq N$, $\theta_{s}:=\left.\operatorname{Ad} u(s)\right|_{N_{\phi}} \in \operatorname{Aut}\left(N_{\phi}\right)$ and $\tau$ be as in $\S 3$.

With $\left(X_{N}, F^{N}\right)$ the smooth flow of weights on $N$, fix a Borel 1-cocycle $c \in Z^{1}\left(F^{N}, K\right)$ having dense range, where $K$ is a locally compact group.

Thanks to [33, Ch. XII, Lemma 3.4], we know that there exists an action $\tilde{\theta}$ of $\mathbb{R}$ on $L^{\infty}(K) \otimes N_{\phi}$ such that: (i) $\tilde{\theta}$ extends the action $\theta$ on $N_{\phi} \cong \mathbb{C} \otimes N_{\phi} \subseteq L^{\infty}(K) \otimes N_{\phi}$; (ii) the restriction of $\tilde{\theta}$ to the center $L^{\infty}(K) \otimes Z\left(N_{\phi}\right)=L^{\infty}\left(K \times X_{N}\right)$ is induced by the skewproduct action of $\mathbb{R}$ derived from $c$. For our purpose, let us recall the construction of the covariant system $\left(L^{\infty}(K) \otimes N_{\phi}, \mathbb{R}, \tilde{\theta}\right)$. First, we consider the $\mathbb{R}$-equivariant disintegration of $\left(N_{\phi}, \mathbb{R}, \theta\right)$ over $\left(X_{N}, \mu_{N}\right)$ :

$$
N_{\phi}=\int_{X_{N}}^{\oplus} N_{\phi}(x) d \mu_{N}(x), \quad \theta_{s} \sim\left\{\theta_{s, x}\right\}, \quad \theta_{s, x}: N_{\phi}(x) \rightarrow N_{\phi}\left(F_{s}^{N} x\right) .
$$

We then have

$$
L^{\infty}(K) \otimes N_{\phi}=\int_{K \times X_{N}}^{\oplus} P(k, x) d\left(m_{K} \times \mu_{N}\right)(k, x),
$$

where $P(k, x)=\mathbb{C} \otimes N_{\phi}(x)$ for any $k \in K$. We put, for any $s \in \mathbb{R}$,

$$
\tilde{\theta}_{s,(k, x)}:=\operatorname{id}_{\mathbb{C}} \otimes \theta_{s, x}: P(k, x) \rightarrow P\left(c(s, x) k, F_{s}^{N} x\right) .
$$

The $*$-automorphism $\tilde{\theta}_{s}$ is then defined, for any

$$
a=\int_{K \times X_{N}}^{\oplus} a(k, x) d\left(m_{K} \times \mu_{N}\right)(k, x) \in L^{\infty}(K) \otimes N_{\phi},
$$


by

$$
\tilde{\theta}_{s}(a)\left(c(s, x) k, F_{s}^{N} x\right):=\tilde{\theta}_{s,(k, x)}(a(k, x))=\left(\operatorname{id}_{\mathbb{C}} \otimes \theta_{s, x}\right)(a(k, x)) .
$$

We also look at the decomposition of the trace $\tau$ :

$$
\tau=\int_{X_{N}}^{\oplus} \tau_{x} d \mu_{N}(x) .
$$

With $\tau_{K}$ the faithful normal trace on $L^{\infty}(K)$ given by integration with respect to $m_{K}$, consider the trace $\tau_{K} \otimes \tau$ on $L^{\infty}(K) \otimes N_{\phi}$. The identity $\tau \circ \theta_{s}=e^{-s} \tau$ yields $\tau_{F_{s}^{N} x} \circ$ $\theta_{s, x}(a(x))=e^{-s} \tau_{F_{s}^{N} x}\left(a\left(F_{s}^{N} x\right)\right)$ for any $a=\int_{X_{N}}^{\oplus} a(x) d \mu_{N}(x) \in N_{\phi}$. By using this, one can check that $\left(\tau_{K} \otimes \tau\right) \circ \tilde{\theta}_{s}=e^{-s}\left(\tau_{K} \otimes \tau\right)$ for all $s \in \mathbb{R}$. Thus, the system $\left(L^{\infty}(K) \otimes N_{\phi}\right.$, $\left.\mathbb{R}, \tilde{\theta}, \tau_{K} \otimes \tau\right)$ is a 'continuous decomposition'. Since $\tilde{\theta}$ acts on the center of $L^{\infty}(K) \otimes N_{\phi}$ ergodically because $c$ has dense range, the crossed product $M:=\mathbb{R}_{\tilde{\theta}^{\ltimes}}\left(L^{\infty}(K) \otimes N_{\phi}\right)$ is an infinite factor. By construction, the smooth flow $\left(X_{M}, F^{M}\right)$ of weights on $M$ is the skew product $K \times{ }_{c} X_{N}$. It is easy to see that $M$, regarded as acting on $L^{2}(\mathbb{R}) \otimes L^{2}(K) \otimes H_{\phi}$, contains the algebra $W^{*}(\mathbb{R}) \otimes \mathbb{C} \otimes \mathbb{C} \vee \pi_{\tilde{\theta}}\left(\mathbb{C} \otimes N_{\phi}\right)\left(=\pi_{\theta}\left(N_{\phi}\right)_{13}\right) \cong \mathbb{R}_{\theta} \ltimes N_{\phi} \cong N$, where $\pi_{\tilde{\theta}}$ is the natural injective $*$-homomorphism from $L^{\infty}(K) \otimes N_{\phi}$ into the crossed product $M$. Let us denote by $v$ the above embedding $N \hookrightarrow M$. Note that, for any $f \in L^{\infty}(K)$, we have

$$
\operatorname{Ad}\left(1 \otimes \rho_{K}(k) \otimes 1\right)\left(\pi_{\tilde{\theta}}(f \otimes 1)\right)=\pi_{\tilde{\theta}}\left(\operatorname{Ad} \rho_{K}(k)(f) \otimes 1\right) .
$$

From this, it follows that $\alpha_{k}:=\left.\operatorname{Ad}\left(1 \otimes \rho_{K}(k) \otimes 1\right)\right|_{M}$ defines an action $\alpha$ of $K$ on $M$. By (C.1), it is clear that the mapping $f \in L^{\infty}(K) \mapsto \pi_{\tilde{\theta}}(f \otimes 1) \in M$ is a $K$-equivariant embedding of the covariant system $\left(L^{\infty}(K), K, \operatorname{Ad} \rho_{K}(\cdot)\right)$ into $(M, K, \alpha)$. Hence, by [28, Ch. II, §2], there exist a coaction $\beta_{0}$ of $K$ on $M^{\alpha}$ and a $*$-isomorphism $\Psi$ from $M$ onto $\widehat{K}_{\beta_{0} \ltimes M^{\alpha}}$ such that $\Psi(a)=\beta_{0}(a)$ for all $a \in M^{\alpha}, \Psi\left(\pi_{\tilde{\theta}}(f \otimes 1)\right)=f \otimes 1$ for all $f \in L^{\infty}(K)$ and $\Psi \circ \alpha_{k}={\widehat{\left(\beta_{0}\right)}}_{k} \circ \Psi$ for all $k \in K$.

LEMMA C.1. The fixed-point algebra $M^{\alpha}$ coincides with $v(N)=\left(\mathbb{R}_{\theta} \ltimes N_{\phi}\right)_{13}$.

Proof. It is easy to see that $v(N)$ is contained in $M^{\alpha}$. By the definition of $\alpha$, we have

$$
\begin{aligned}
M^{\alpha} & =M \cap\left\{1 \otimes \rho_{K}(k) \otimes 1: k \in K\right\}^{\prime}=M \cap\left(B\left(L^{2}(\mathbb{R})\right) \otimes W^{*}(K) \otimes B\left(H_{\phi}\right)\right) \\
& \subseteq\left(B\left(L^{2}(\mathbb{R})\right) \otimes L^{\infty}(K) \otimes N_{\phi}\right) \cap\left(B\left(L^{2}(\mathbb{R})\right) \otimes W^{*}(K) \otimes B\left(H_{\phi}\right)\right) \\
& =B\left(L^{2}(\mathbb{R})\right) \otimes \mathbb{C} \otimes N_{\phi} .
\end{aligned}
$$

So let us suppose that $X$ is in $B\left(L^{2}(\mathbb{R})\right) \otimes N_{\phi}$ such that $X_{13} \in M^{\alpha}$. Since $\left.\tilde{\theta}_{t}\right|_{\mathbb{C} \otimes N_{\phi}}=\operatorname{id} \otimes \theta_{t}$ for all $t \in \mathbb{R}$, we have

$$
X_{13}=\left(\operatorname{Ad} \lambda_{\mathbb{R}}(-t) \otimes \tilde{\theta}_{t}\right)\left(X_{13}\right)=\left(\operatorname{Ad} \lambda_{\mathbb{R}}(-t) \otimes \mathrm{id} \otimes \theta_{t}\right)\left(X_{13}\right) .
$$

This shows that $X$ belongs to $\left(B\left(L^{2}(\mathbb{R})\right) \otimes N_{\phi}\right)^{\mathrm{Ad} \lambda_{\mathbb{R}}(-\cdot) \otimes \theta}=\mathbb{R}_{\theta} \ltimes N_{\phi}$. Therefore, $X_{13}$ is in $v(N)$.

LEMMA C.2. The fixed-point algebra $\left(M^{\alpha}\right)^{\beta_{0}}$ of $\beta_{0}$ contains the algebra $v\left(N_{\phi}\right)=$ $\pi_{\theta}\left(N_{\phi}\right)_{13}$. 
Proof. Clearly, $\pi_{\theta}(a)_{13}=\pi_{\tilde{\theta}}(1 \otimes a)$ commutes with $\pi_{\tilde{\theta}}(f \otimes 1)$ for any $a \in N_{\phi}$ and any $f \in L^{\infty}(K)$. Through the isomorphism $\Psi$, this is equivalent to saying that $\beta_{0}(a)$ commutes with $f \otimes 1$. From this, we find that $\beta_{0}\left(\pi_{\theta}(a)_{13}\right)=1 \otimes \pi_{\theta}(a)_{13}$ for any $a \in N_{\phi}$.

Thanks to Lemma C.1, the equation $\beta_{1}:=\left(\mathrm{id} \otimes v^{-1}\right) \circ \beta_{0} \circ v$ defines a coaction $\beta_{1}$ of

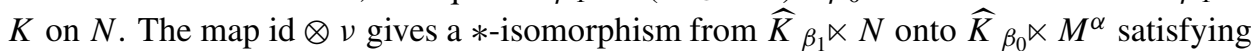
$(\mathrm{id} \otimes v)\left(\beta_{1}(a)\right)=\beta_{0}(v(a))$ for all $a \in N$ and $\left.\widehat{\left(\beta_{0}\right)}\right)_{k} \circ(\mathrm{id} \otimes v)=(\mathrm{id} \otimes \nu) \circ{\widehat{\left(\beta_{1}\right.}}_{k}$ for all $k \in K$.

By Lemma C.2, the fixed-point algebra $N^{\beta_{1}}$ contains $N_{\phi}$. By Theorem 4.1, there is a cocycle $c_{1} \in Z^{1}\left(F^{N}, K\right)$ such that $\beta_{1}$ is the extended modular coaction $\beta_{c_{1}}^{\phi}$ associated with $c_{1}$. Since $(M, K, \alpha)$ is conjugate to $\left(L^{\infty}(K) \otimes_{c_{1}} N, K, \widehat{\left(\beta_{c_{1}}^{\phi}\right)}\right)$, it follows that $c_{1}$ is cohomologous to $c$, and hence we may identify the covariant system $(M, K, \alpha)$ obtained above with the skew product $\left(L^{\infty}(K) \otimes_{c} N, K, \widehat{\left(\beta_{c}^{\phi}\right)}\right)$.

By looking carefully at the isomorphisms $\Psi$ and id $\otimes v$ obtained above, we deduce the following.

Proposition C.3. The covariant system $\left(L^{\infty}(K) \otimes N_{\phi}, \mathbb{R},\left.\operatorname{Ad} \beta_{c}^{\phi}(u(s))\right|_{L^{\infty}(K) \otimes N_{\phi}}\right.$, $\left.\tau_{K} \otimes \tau\right)$ gives a continuous decomposition of the skew-product algebra $L^{\infty}(K) \otimes_{c} N$. Therefore, the smooth flow of weights on $L^{\infty}(K) \otimes_{c} N$ is the covariant system $\left(L^{\infty}(K) \otimes\right.$ $\left.Z\left(N_{\phi}\right), \mathbb{R}, \operatorname{Ad} \beta_{c}^{\phi}(u(\cdot))\right)$, as proven in $\S 6$.

Corollary C.4. Let $\left(L^{\infty}(K) \otimes N_{\phi}, \mathbb{R},\left.\operatorname{Ad} \beta_{c}^{\phi}(u(s))\right|_{L^{\infty}(K) \otimes N_{\phi}}, \tau_{K} \otimes \tau\right)$ be as in Proposition C.3. Also, let $\psi$ denote the dominant weight on $L^{\infty}(K) \otimes_{c} N$ obtained as the dual weight of $\tau_{K} \otimes \tau$. Then $\psi$ is proportional to the dual weight $\tilde{\phi}$ (of $\phi$ ) on $L^{\infty}(K) \otimes_{c} N=\widehat{K}_{\beta_{c}^{\phi}} \ltimes N$. In particular, $\tilde{\phi}$ is always dominant.

Proof. Before we proceed to a proof, let us note that, if $L^{\infty}(K) \otimes_{c} N$ is of type $I I I$, then it is easy to see that $\tilde{\phi}$ is dominant, because $c \tilde{\phi}$ is clearly equivalent to $\tilde{\phi}$ for any $c>0$ due to the fact that $\phi$ itself is by assumption dominant and $\tilde{\phi}=\phi \circ T_{\alpha}$, where $\alpha$ is the dual action of $\beta_{c}^{\phi}$.

Let $\omega:=\tilde{\phi}$ and $M:=L^{\infty}(K) \otimes_{c} N$. By Proposition 3.8 and [39, Lemma 1.2], we have $\sigma_{t}^{\omega}(f \otimes 1)=f \otimes 1$ for all $f \in L^{\infty}(K)$ and all $t \in \mathbb{R}$. From this, we see that the centralizer $M_{\omega}$ of $\omega$ contains $L^{\infty}(K) \otimes \mathbb{C} \vee \beta_{c}^{\phi}\left(N_{\phi}\right)=L^{\infty}(K) \otimes N_{\phi}$. Let $u_{t}:=(D \omega: D \psi)_{t}$, the Connes cocycle derivative of $\omega$ with respect to $\psi$. Since both $M_{\omega}$ and $M_{\psi}$ contains $L^{\infty}(K) \otimes N_{\phi}$, it follows from the relative commutant theorem that each $u_{t}$ belongs to $M_{\psi}^{\prime} \cap M=Z\left(M_{\psi}\right)=L^{\infty}\left(K \times X_{N}\right)$. In particular, $u_{t}$ is a one-parameter unitary group.

For any $s, t \in \mathbb{R}$, we have, by [35, Proposition 3.7],

$$
\sigma_{t}^{\omega}\left(\beta_{c}^{\phi}(u(s))\right)=\beta_{c}^{\phi}\left(\sigma_{t}^{\phi}(u(s))\right)=\beta_{c}^{\phi}\left(e^{-i s t} u(s)\right)=e^{-i s t} \beta_{c}^{\phi}(u(s)) .
$$

In the meantime, by Proposition C.3, we also have $\sigma_{t}^{\psi}\left(\beta_{c}^{\phi}(u(s))\right)=e^{-i s t} \beta_{c}^{\phi}(u(s))$. Hence, each $u_{t}$ is contained in $\left(L^{\infty}(K) \otimes L^{\infty}\left(X_{N}\right)\right)^{\operatorname{Ad} \beta_{c}^{\phi}(u(\cdot))}$, which is the scalar multiples of the identity by Proposition C.3. Hence, there is a constant $c>0$ such that $u_{t}=c^{i t}$. This means that $\omega=c \psi$. 
D. Appendix. Existence of Borel 1-cocycles on properly ergodic flows having dense range The following is an easy generalization of [22, Proposition A.5] to the noncompact case. We need to assume below that the locally compact group $K$ is amenable, because of [41, Theorems 2.1 and 3.1].

PROPOSITION D.1. Let $(X, \mu)$ be a properly ergodic $\mathbb{R}$-space. Then, for any amenable locally compact group $K$, there exists a Borel 1-cocycle $c: \mathbb{R} \times X \rightarrow K$ having dense range.

Proof. By representing the given flow as a flow built under a ceiling function with an ergodic single transformation (cf. [33, Ch. XII, §3, Theorem 3.2]), it suffices to prove the proposition for a $\mathbf{Z}$-action. Let $S$ be a given non-singular and properly ergodic transformation on $(X, \mu)$. We show that there exists a Borel 1-cocycle $c: \mathbf{Z} \times X \rightarrow K$ having dense range. We take a type $I I_{1}$ ergodic transformation $T$ on a standard Borel probability space $(Y, v)$. Thanks to [15], [21] or [1], there exists a Borel 1-cocycle $c^{\prime}$ : $\mathbf{Z} \times Y \rightarrow K$ which has dense range in $K$. From this point on, we have only to follow the argument in the proof of [22, Proposition A.5] in order to obtain the result.

Acknowledgements. The author thanks Professor Aoi very much for letting him know about his preprint [2], where he independently constructed the skew-product algebra out of a Borel 1-cocycle on an ergodic $\mathbb{R}$-space. Although the results of Izumi [22] seem to serve as a major motivation of Aoi's work as well, we emphasize that his construction is different from ours. The author is also grateful to Professor Izumi for an interesting discussion at an early stage of this work. Finally, the author thanks the referee for pointing out a flaw of arguments in the original manuscript.

\section{REFERENCES}

[1] J. Aaronson and B. Weiss. On Herman's theorem for ergodic, amenable group extensions of endomorphisms. Ergod. Th. \& Dynam. Sys. 24 (2004), 1283-1293.

[2] H. Aoi. A characterization of coactions by their dual actions. Submitted.

[3] H. Aoi and T. Yamanouchi. A characterization of coactions whose fixed-point algebras contain special maximal abelian $*$-subalgebras. Ergod. Th. \& Dynam. Sys. to appear.

[4] D. L. Armacost. The Structure of Locally Compact Abelian Groups (Monographs and Textbooks in Pure and Applied Mathematics, 68). Marcel Dekker, New York, 1981.

[5] A. Connes. Une classification des facteurs de type III. Ann. Sci. École Norm. Sup. (4) 6 (1973), $133-252$.

[6] A. Connes. On hyperfinite factors of type $\mathrm{III}_{0}$ and Krieger's factors. J. Funct. Anal. 18 (1975), 318-327.

[7] A. Connes. Outer conjugacy classes of automorphisms of factors. Ann. Sci. École Norm. Sup. (4) 8 (1975), 383-419.

[8] A. Connes. Classification of injective factors. Cases $\mathrm{II}_{1}, \mathrm{II}_{\infty}, \mathrm{III}_{\lambda}, \lambda \neq 1$. Ann. of Math. (2) 104 (1976), 73-115.

[9] A. Connes and M. Takesaki. The flow of weights of factors of type III. Tohoku Math. J. 29 (1977), 473-575.

[10] P. Eymard. L'algèbre de Fourier d'un groupe localement compact. Bull. Soc. Math. France 92 (1964), 181-236.

[11] R. C. Fabec. Normal ergodic actions and extensions. Israel J. Math. 40 (1981), 175-186.

[12] R. C. Fabec. Cocycles, extensions of group actions, and bundle representations. J. Funct. Anal. 56 (1984), 79-98. 
[13] J. Feldman and C. C. Moore. Ergodic equivalence relations, cohomology and von Neumann algebras, I. Trans. Amer. Math. Soc. 234 (1977), 289-324.

[14] J. Feldman and C. C. Moore. Ergodic equivalence relations, cohomology and von Neumann algebras, II. Trans. Amer. Math. Soc. 234 (1977), 325-359.

[15] V. Y. Golodets and S. D. Sinelshchikov. Locally compact groups appearing as ranges of cocycles of ergodic $\mathbb{Z}$-actions. Ergod. Th. \& Dynam. Sys. 5 (1985), 47-57.

[16] U. Haagerup. Operator valued weights in von Neumann algebras, I. J. Funct. Anal. 32 (1979), 175-206.

[17] U. Haagerup. Operator valued weights in von Neumann algebras, II. J. Funct. Anal. 33 (1979), 339-361.

[18] U. Haagerup. Connes' bicentralizer problem and uniqueness of the injective factor of type $\mathrm{III}_{1}$. Acta Math. 158 (1987), 95-148.

[19] U. Haagerup and E. Størmer. Equivalence of normal states on von Neumann algebras and the flow of weights. Adv. Math. 83 (1990), 180-262.

[20] T. Hamachi and M. Osikawa. Ergodic groups of automorphisms and Krieger's theorems. Proc. 3rd Seminar on Mathematical Science (Keio University, 1981).

[21] R. S. Ismagilov. Application of the group algebra of the problem of the tail $\sigma$-algebra of a random walk on a group and the problem of ergodicity of a skew-product action. Math. USSR Izv. 31 (1988), 209-222.

[22] M. Izumi. Canonical extension of endomorphisms of type III factors. Amer. J. Math. 125 (2003), 1-56.

[23] M. Izumi, R. Longo and S. Popa. A Galois correspondence for compact groups of automorphisms of von Neumann algebras with a generalization to Kac algebras. J. Funct. Anal. 155 (1998), 25-63.

[24] W. Krieger. On ergodic flows and the isomorphism of factors. Math. Ann. 223 (1976), 19-70.

[25] J. Kustermans and S. Vaes. Locally compact quantum groups in the von Neumann algebraic setting. Math. Scand. 92 (2003), 68-92.

[26] S. A. Morris. Pontryagin Duality and the Structure of Locally Compact Abelian Groups (London Mathematical Society Lecture Note Series, 29). Cambridge University Press, Cambridge 1977.

[27] Y. Nakagami. Essential spectrum $\Gamma(\beta)$ of a dual action on a von Neumann algebra. Pacific J. Math. 70 (1977), 437-479.

[28] Y. Nakagami and M. Takesaki. Duality for Crossed Products of von Neumann Algebras (Lecture Notes in Math, 731). Springer, Berlin, 1979.

[29] W. Rudin. Fourier Analysis on Groups. Wiley, New York, 1962.

[30] J.-L. Sauvageot. Image d'un homomorphisme et flot des poids d'une relation d'équivalence mesurée. Math. Scand. 42 (1978), 71-100.

[31] Ş. Strătilă. Modular Theory in Operator Algebras. Abacus Press, Boston, MA, 1981.

[32] M. Takesaki. Conditional expectations in von Neumann algebras. J. Funct. Anal. 9 (1972), 306-321.

[33] M. Takesaki. Theory of Operator Algebras II (Encyclopaedia of Mathematical Sciences, 125). Springer, Berlin.

[34] M. Takesaki and N. Tatsuuma. Duality and subgroups. Ann. of Math. (2) 98 (1971), 344-364.

[35] S. Vaes. The unitary implementation of a locally compact quantum group action. J. Funct. Anal. 180 (2001), 426-480.

[36] S. Vaes. Strictly outer actions of groups and quantum groups. J. reine angew. Math. 578 (2005), $147-184$.

[37] S. Vaes and L. Vainerman. Extensions of locally compact quantum groups and the bicrossed product construction. Adv. Math. 175 (2003), 1-101.

[38] T. Yamanouchi. Galois groups of quantum group actions and regularity of fixed-point algebras. Trans. Amer. Math. Soc. 355 (2003), 2813-2828.

[39] T. Yamanouchi. Canonical extension of actions of locally compact quantum groups. J. Funct. Anal. 201 (2003), 522-560.

[40] R. J. Zimmer. Extension of ergodic group actions. Illinois J. Math. 20 (1976), 373-409.

[41] R. J. Zimmer. Amenable ergodic group actions and an application to Poisson boundaries of random walks. J. Funct. Anal. 27 (1978), 350-372.

[42] R. J. Zimmer. Ergodic Theory and Semisimple Groups. Birkhäuser, Basel, 1984. 